\title{
Wireless Cloud Networks for the Factory of Things: Connectivity Modeling and Layout Design
}

\author{
Stefano Savazzi, Member, IEEE, Vittorio Rampa, Umberto Spagnolini, Member, IEEE
}

\begin{abstract}
Large-scale adoption of dense cloud-based wireless network technologies in industrial plants is mandatorily paired with the development of methods and tools for connectivity prediction and deployment validation. Layout design procedures must be able to certify the quality (or reliability) of network information flow in industrial scenarios characterized by harsh propagation environments. In addition, these must account for possibly coexisting heterogeneous radio access technologies as part of the internet of things (IoT) paradigm, easily allow postlayout validation steps, and be integrated by industry standard CAD-based planning systems. The goal of the paper is to set the fundamentals for comprehensive industry-standard methods and procedures supporting plant designer during wireless coverage prediction, virtual network deployment and post-layout verification. The proposed methods carry out the prediction of radio signal coverage considering typical industrial environments characterized by highly dense building blockage. They also provide a design framework to properly deploy the wireless infrastructure in interference-limited radio access scenarios. In addition, the model can be effectively used to certify the quality of machine type communication by considering also imperfect descriptions of the network layout. The design procedures are corroborated by experimental measurements in an oil refinery site (modelled by 3D CAD) using industry standard ISA IEC 62734 devices operating at $2.4 \mathrm{GHz}$. A graph-theoretic approach to node deployment is discussed by focusing on practical case studies, and also by looking at fundamental connectivity properties for random deployments.
\end{abstract}

\section{INTRODUCTION}

The adoption of wireless communication and sensor networks in the industrial context is becoming of strategic interest for manufacturers and plant designers [1]. As an example, when increasing the production of existing industrial plants, wireless systems provides a cost-effective solution to complement the existing wired network and thus augment monitoring without the costly (and often unfeasible for logistic reasons) re-wiring over the existing plants. Compared to wired systems, wireless technologies have the advantage of low cost, mobility and energy-efficiency, compactness, and flexibility to overcome obstacles more easily than existing wired solutions.

The work has been supported partially by the European Research Project DI WI NE(Dense Cooperative Wireless Cloud Network) under FP7 ICT Objective 1.1 - The Network of the Future, and by Saipem, a subsidiary of eni S.p.A.

S. Savazzi and V. Rampa are with the IEIITInstitute of the Italian National Research Council (CNR), c/o Politecnico di Milano P.zza L. da Vinci 32, I-20133 Milano, Italy (e-mail: stefano.savazzi, vittorio.rampa@ieiit.cnr.it).

U. Spagnolini, is with the Dipartimento di Elettronica, I nformazione e Bioingegneria (DEIB), Politecnico di Milano, P.zza L. da Vinci 32, I-20133 Milano, Italy (e-mail: umberto.spagnolini@polimi.it).

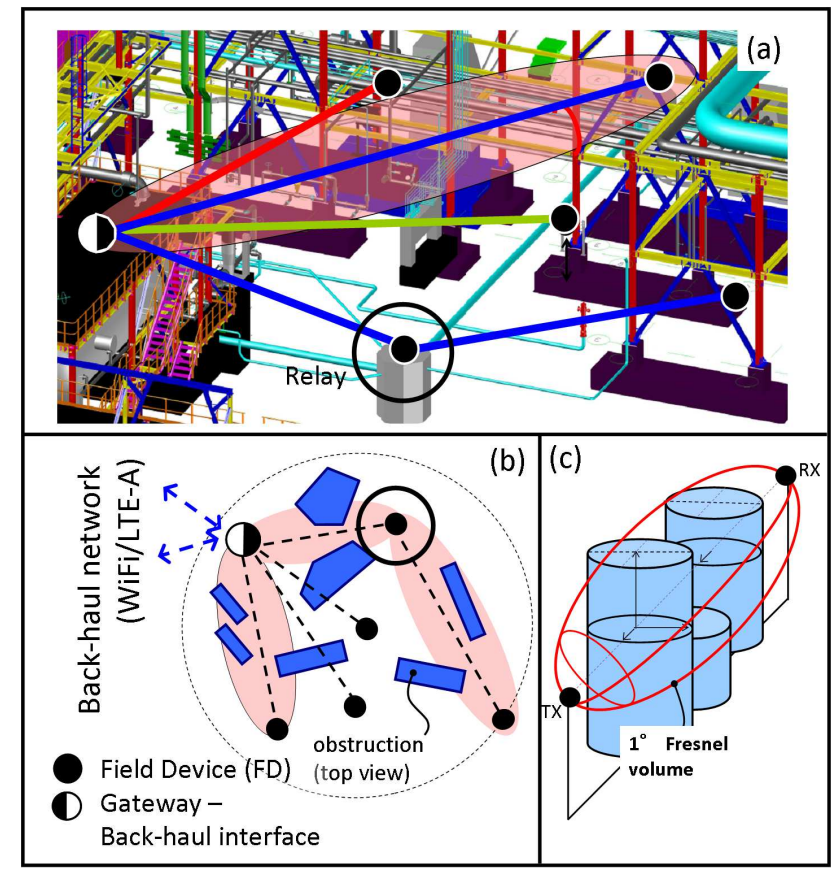

Fig. 1. Industrial wireless sensor network architecture. Field device (FD) layout over 3D-CAD model of the plant (oil refinery site). Bottom figure: cloud-based industrial wireless sensor network architecture with Gateways serving as WiFi enabled cloud access nodes. Short-range machine type wireless communication is subject to highly dense buidling blockage (LOS/NLOS type links).

These benefits come without additional maintenance efforts as wireless devices have now battery lifetime comparable to the planned maintenance schedules of wired systems.

Next generation wireless sensor network technologies are expected to be integrated into the Internet of Things (IoT) paradigm [2], allowing for the global interconnection of heterogeneous smart physical objects with advanced functionalities. Following the paradigm of IoT, emerging technologies inside modern factories are evolving to intelligent environments or "Factory of Things (FoT)" [3], where wireless network technologies and mobile information access are now playing a key role for the efficient design of industrial processes.

Cloud-enabled wireless industrial sensor networks [2]-[5] is an emerging paradigm in machine-type communication: it consists of self-organizing, massively dense air-interacting wireless devices (serving as sensors, actuators, relays or distributed computing nodes) that have the ability to gather and process pervasive information about the state of the industrial plant, by enabling early detection and localization [6] of any dan- 
gerous situation. Cloud-based sensor networks have recently attracted the interest of several research fields ranging from ubiquitous computing, machine learning, information theory [5], and social networking [7]. Sensor-cloud architectures are designed to integrate cloud computing functionalities into the wireless monitoring and control network infrastructure, allowing for pervasive, collective and distributed computation of data by end-users, supporting different industrial applications [4]. According to this view, sensor and actuator devices do not simply serve as possibly faulty end-points but also as critical computing and storage resources that must be reliably connected to satisfy tight quality-of-service (QoS) constraints.

Although many technological solutions and standards (e.g., WiFi/LTE, IEC standards WirelessHART, ISA100.11a [8]) have been investigated for application-specific contexts, even if the proposed solutions effectively address consumer needs, they are not yet ready for cloud-enabled industrial applications with high safety, reliability, security and real-time requirements [3]. This is also due to the limitations of existing network design procedures that are not suitable to address network optimization in complex and harsh environments. In addition, industrial layouts are characterized in many cases by multiple coexisting heterogeneous networks, as part of the FoT architecture. Industry-standard design procedures for wireless cloud network optimization must be therefore able to certify the reliability and safety of radio links under harsh conditions. Moreover, these procedures must be supported by various industrial planning tools [9] (CAD, CAE, CAM) in order to achieve a seamless planning, design and operation environment, regardless of the wireless/wired communication technology employed. Developing consistent design methodologies for (possibly heterogeneous) industrial wireless networks is also becoming mandatory as supporting tool for the technology suppliers/vendors during every phase of system set-up, precommissioning, integration, and post-layout testing.

Purpose of this paper is to make a first and unique attempt towards the proposal of algorithms and the definition of industry-compliant tools able to support virtual coverage prediction, layout design and deployment of industrial wireless sensor networks for cloud-based applications and decentralized architectures. Experimental measurements are carried out to evaluate the effectiveness of the proposed toolkit based on industry standard devices operating at $2.4 \mathrm{GHz}$. The IEEE 802.15.4 physical (PHY) layer is considered in this paper being the standard choice for IEC 62591 (WirelessHART) and IEC 62734 (ISA SP100) compliant wireless field instruments [8].

\section{A. Wireless Cloud Networks for machine-type communication}

The cloud-based FoT network architecture considered in this paper consists of dense, massively interacting groups of wireless nodes that cooperate to provide seamless communication services to user terminals. The architecture is promising as it can support decentralized consensus-based techniques for joint estimation of key parameters, digital and analog network coding [5] in which field devices (FDs), acting as sensors, actuators or relaying nodes, can forward a jointly coded combination of incoming data streams, implementing a virtual multiple antenna system [10]. In order to enable those advanced functionalities, the physical structure of the network must be optimized to certify the quality/reliability of the information flow, and to guarantee fast processing and convergence of any decentralized task.

The $3 \mathrm{D}$ view of the testing site considered as example in this paper is illustrated in Fig. 1: data originated from wireless FDs are 'flooded' throughout the network toward Gateway (GW) devices. The GWs have the role of cloud access nodes (e.g., for fixed/mobile WiFi enabled users), connecting the FDs to a backbone wired/wireless network (Fieldbus/LTE), and providing a uniform access interface to devices outside the cloud.

\section{B. Contributions}

Contributions of the paper are summarized as follows.

- A novel stochastic model is proposed to describe shortrange machine-type wireless connectivity in industrial environments characterized by highly dense building blockage. This prediction model is applicable to different industrial settings and relevant scenarios (where obstructions are fixed or predictable). It can be effectively used to certify the link reliability accounting for errors during on-site positioning of instruments and imperfect/inaccurate description of the 3D environment that might occur throughout the various industrial plant design phases. A 3D CAD software tool is used to identify the structure of the building blockage [11] from which the reliability of the radio link can be predicted using the proposed model. Based on well consolidated principles from propagation and diffraction theory (revised in Sect. 2), the model allows for the prediction (before on-site deployment) of the link quality information (LQI) for short-range machine-tomachine communication based on the positions of the industrial equipment and the configuration of the obstructions (Sect. 3). Model accuracy is validated by measurement campaigns from large-size oil refinery plants. Post-layout verification is carried out by exploiting the prediction model along with test positions verifications.

- FoT enabling wireless cloud technologies are expected to operate inside the factory over unlicensed $2.4 \mathrm{GHz}$, these coexist with other devices employing different radio protocols. The problem of modeling the coexistence between WiFi and IEEE 802.15.4 networks in critical high traffic load scenarios is addressed in Sect. 4. Based on several experimental tests, the impact of different IEEE 802.15.4 PHY configurations, WiFi traffic loads and interference overlapping on coexistence are also discussed.

- The design of cloud connectivity (Sect. 5) is discussed based on a graph-theoretic approach and evaluated by focusing on practical case studies. The deployment of relays is adopted to improve the reliability of weakly connected networks characterized by a high percentage of unreliable links. Weakly connected clusters are identified according to a spectral graph partitioning method, and relays are planned accordingly. Practical deployment case studies validate the effectiveness and the accuracy of the proposed model. To provide a comprehensive 
performance evaluation, in Sect. 6 the cloud network is modeled as a random geometric graph, while connectivity, energy consumption and interference coexistence are analyzed. The heterogeneity of machine-type links (in terms of configuration of obstructions) suggests relevant conditions on propagation for which "small-world" connectivity phenomena [12] emerge, thus simplifying relay placement and topology control.

\section{MODELING OF SHORT-RANGE PROPAGATION}

This section introduces the modeling of the short-range channel, being the first step for the prediction of the wireless machine-type connectivity and coverage. Although several models have been proposed in the literature, (e.g., see [13] for reviewing) these cannot fully capture the unique propagation characteristics of industrial environments. In addition, most of conventional ray-tracing tools turn out not to be practical to process the high number of structures and their complexity in large industrial sites [14]. This motivates the development of accurate site-specific channel models based on a set of measurements taken in typical industrial sites. The proposed wireless propagation model describes the correlation between the size and the locations of the objects obstructing the lineof-sight (LOS) path (or Fresnel volumes) and the reliability of the wireless link in terms of received signal strength (RSS). For any link $\ell$ connecting a pair of FDs at distance $d$, the RSS $\gamma_{\ell}$ is modeled as

$$
\gamma_{\ell}=\underbrace{g_{0}(d) \cdot \sigma_{\ell}^{-1}}_{\text {LQI: } g_{\ell}(d)} s_{\ell}+n_{\ell} .
$$

It combines three terms: $i)$ a static component $\mathrm{E}\left[\gamma_{\ell}\right]=g_{\ell}(d)$ characterized by a distance dependent $(d)$ LOS term $g_{0}(d)$ and a link-specific excess attenuation $\sigma_{\ell}$ due to propagating wavefronts diffracting around the building blockage; ii) a dynamic random component $s_{\ell}$ due to moving people [6] or field equipment, typically modeled by Rician distribution [13] with $\mathrm{E}\left[s_{\ell}\right]=1$; iii) random disturbance $n_{\ell}$ due to noise and cochannel interference with power $\mathrm{E}\left[n_{\ell}\right]=\mu_{\ell}$. In what follows, we focus on modeling of the static component $g_{\ell}(d)$ being a practical indicator of link quality, also referred to as LQI [15]. The impact of dynamic component $s_{\ell}$ and disturbance $n_{\ell}$ in heterogeneous network scenarios are assessed in Sect. 4 according to experiments in real environments.

As typically assumed in recent standardization bodies such as ITU [13], the distance-dependent static component $g_{0}(d)$ provides a description of the radio signals reflecting from the (flat) terrain, while in short-range propagation ${ }^{1}$, the obstruction $\sigma_{\ell}$ caused by building blockage acts as an additive (in $\mathrm{dB}$ scale) and independent term. The term $g_{0}(d)$ can be described in terms of the Fresnel distance $d_{F}$ :

$$
\left[g_{0}(d)\right]_{d B}=\left\{\begin{array}{cc}
{\left[g_{0}\left(d_{0}\right)\right]_{d B}-\alpha_{0} \times\left[d / d_{0}\right]_{d B}} & d \leq d_{F} \\
{\left[g_{0}\left(d_{F}\right)\right]_{d B}-\alpha_{F} \times\left[d / d_{F}\right]_{d B},} & d>d_{F}
\end{array}\right.
$$

where the operator $[\cdot]_{d B}=10 \log _{10}(\cdot)$ indicates $\mathrm{dB}$ scale conversion. The component is modeled as a function of the path-loss exponents $\alpha_{0}, \alpha_{F}$ (see [13]) for $d \leq d_{F}$ and $d>$

\footnotetext{
${ }^{1}$ below $500 \mathrm{~m}$ in outdoor environments (typical for micro-cells).
}

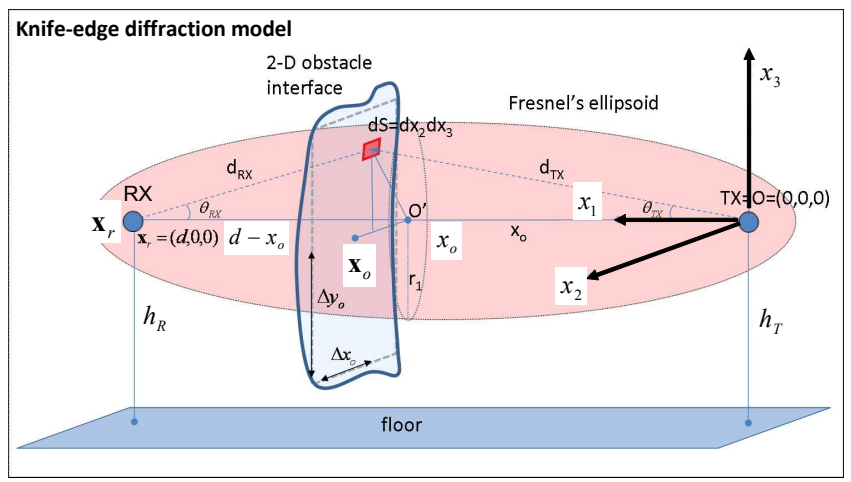

Fig. 2. Knife-edge diffraction model for random surface

$d_{F}$, respectively, while $g_{0}\left(d_{0}\right)$ is the free space channel gain measured at a reference distance (here $d_{0}=2 \mathrm{~m}$ ). The Fresnel distance $d_{F}=2 h_{R} h_{T} / \lambda$ depends on the wavelength $\lambda$ and on the antennas height from the ground of the transmitter $h_{T}$ and the receiver $h_{R}$. The path loss exponent can be reasonably set to $\alpha_{0}=2$ for $d \leq d_{F}$ in short range environments where ground reflections can be neglected. Larger exponents $\alpha_{F}>\alpha_{0}$ (with typically $2 \leq \alpha_{F} \leq 3$ ) are caused by reflections from the ground and are observed over longer range for $d>d_{F}$.

Diffraction theory provides an effective tool for modeling the signal power excess attenuation $\sigma_{\ell}$ as due to objects obstructing the Fresnel zones [16] and absorbing fractions of the propagating energy of the wavefield. The mathematical framework instrumental to the evaluation of diffraction loss $\sigma_{\ell}$ for a given configuration of the obstruction is reviewed in Sect. 2.A.

\section{A. Modeling of attenuation constrained by building blockage}

Herein, we model the additional attenuation $\sigma_{\ell}$ in (1) as due to obstacles acting as perfectly absorbing interfaces. The model for the prediction of the building blockage term $\sigma_{\ell}$ is based on the Fresnel-Kirchhoff method. The Huygen's principle for propagating wavefield [16] is used to predict the actual field strength diffracted by obstacles modeled as knifeedge objects. In spite of the simplicity of this method, in Sect. 3-4 it is shown to be accurate enough for wireless link quality prediction. The attenuation $\sigma_{\ell}(1)$ is inferred from the received electric field as

$$
\sigma_{\ell}=\left|E_{0} / E_{1}\right|^{2}
$$

where the ratio $E_{0} / E_{1}$ describes the obstruction loss in excess with respect to the free space electric field $E_{0}$ (without obstructions). The electric field $E_{1}$ at the receiver can be interpreted as generated by a virtual array of Huygens' sources located in the target plane. Large-size metallic objects obstructing the wireless link absorb a large amount of the signal intensity and limit the received field to a small fraction of the one that would be observed under free-space propagation (being $\left|E_{1} / E_{0}\right| \ll 1$ ).

According to the simplified description of the layout geometry illustrated in Fig. 2, the radio link is characterized 
by a transmitter placed for convenience in the origin $O$ of the axes $\left(x_{1}, x_{2}, x_{3}\right)$ and the receiver at distance $d$ with coordinates $\mathbf{x}_{r}=[d, 0,0]$. The single obstruction is modeled as a knife-edge $2 \mathrm{D}$ object in the $\left(x_{2}, x_{3}\right)$ plane with size described by vector $\Delta \mathbf{x}_{o}$ and barycenter located at coordinates $\mathbf{x}_{o}=\left[x_{o}, y_{o}, 0\right]$ in the surroundings of the direct LOS path (i.e., at distance $y_{0}>0$ from the LOS). The object occupies an obstructing surface $\left(x_{2}, x_{3}\right) \in \mathcal{S}\left(\mathbf{x}_{r}, \mathbf{x}_{o} ; \Delta \mathbf{x}_{o}\right)$. The field intensity $E_{1}$ at the receiver can be written in general as

$$
E_{1}\left(\mathbf{x}_{r}, \mathbf{x}_{o} ; \Delta \mathbf{x}_{o}\right)=E_{0}-\int_{\left(x_{2}, x_{3}\right) \in \mathcal{S}\left(\mathbf{x}_{r}, \mathbf{x}_{o} ; \Delta \mathbf{x}_{o}\right)} d E
$$

where $d E$ is the electric field that is subtracted due to diffraction effects and caused by the elementary Huygen' source over the area $d x_{2} d x_{3}$. The model (4) for a single obstacle can be recursively applied and extended to multiple obstacles by following the Deygout approach (see [11] for details). Considering antennas with omnidirectional pattern at both sides, and since the object responsible for the most significant attenuation can be reasonably assumed as located in the surroundings of the direct path, the electric field $d E$ can be approximated [17] for $x_{2}, x_{3}, \lambda \ll x_{o}, x_{r}-x_{o}$ as

$$
d E=\frac{1}{r_{1}^{2}\left(\mathbf{x}_{r}, \mathbf{x}_{o}\right)} j E_{0} \exp \left[-j \pi \frac{x_{2}^{2}+x_{3}^{2}}{r_{1}^{2}\left(\mathbf{x}_{r}, \mathbf{x}_{o}\right)}\right] d x_{2} d x_{3}
$$

with $r_{1}\left(\mathbf{x}_{r}, \mathbf{x}_{o}\right)=\sqrt{\lambda x_{o}\left(d-x_{o}\right) \times d^{-1}}$ being the radius of the first Fresnel's zone circular section [16] corresponding to the location of the obstruction (at distance $x_{o}$ from the transmitter). The solution of (4) is obtained by numerical integration for arbitrary complex obstructing surfaces $\mathcal{S}$. Approximated solutions are also available in some simplified settings, see [17] and [11].

\section{B. Diffraction loss from $2 \mathrm{D}$ rectangular obstructions $(2.4 \mathrm{GHz})$}

To gain further insight into the relationship between the obstruction size and the corresponding field loss, in Fig. 3(a) we focus on a reference scenario (considered also in Sect. 3) where the obstruction is characterized by a knifeedge object with rectangular cross-section having $\Delta \mathbf{x}_{o}=$ $\left(0, \Delta x_{o}, \Delta y_{o}\right)$, lateral half-dimension $\Delta x_{o}$ and unbounded vertical half-dimension $\left(\Delta y_{o} \rightarrow \infty\right)$ sometimes referred to as $2.5 \mathrm{D}$ obstacle $^{2}$. In the reference example, the object is located halfway at distance $x_{o}=d / 2$ from the transmitter and at distance $y_{o}$ from the direct path. The excess attenuation $\sigma_{\ell}$ (in dB scale) due to diffraction is evaluated in Fig. 3-(b) for varying lateral size $\Delta x_{o}=0 \div 3 r_{1}$ and object positions $y_{o}=0 \pm 3 r_{1}$ to model arbitrary obstruction configurations. As an example, a wireless link of distance $d=30 \mathrm{~m}$, with $\lambda=0.12 m(2.4 G H z$ ISM Band) and equipped with omnidirectional antennas) would experience a Fresnel radius of $r_{1}=1.4 \mathrm{~m}$, while the rectangular obstruction located at $x_{o}=15 \mathrm{~m}$ from the transmitter has horizontal half-dimension $\Delta x_{o}<3 \times r_{1}=4.2 \mathrm{~m}$. Three relevant reference obstruction configurations can be highlighted: $i)$ the unobstructed direct LOS path (i.e., for $\Delta x_{o}<y_{o}$ ) with corresponding

\footnotetext{
${ }^{2}$ Vertical half-dimension can be assumed in practice as reasonably high $\Delta y_{o} \gg r_{1}$ to model typical obstructions caused by large equipment units and buildings
}

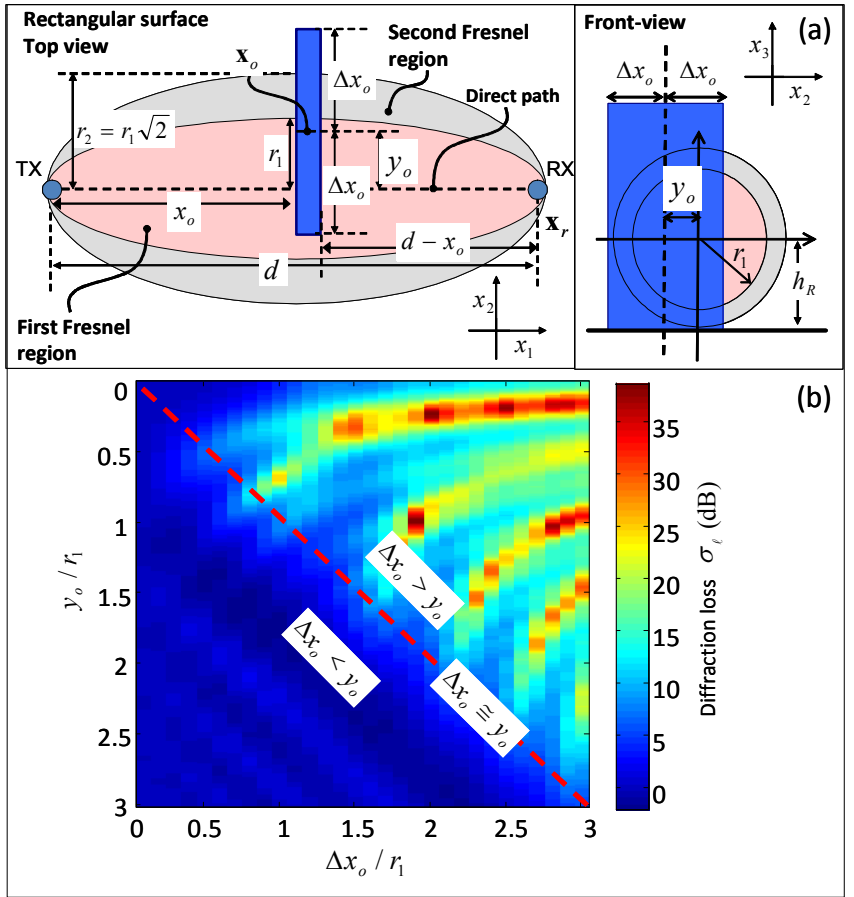

Fig. 3. Knife-edge diffraction model for rectangular (vertical unbounded) surface (a). Diffraction loss for varying position $\left(y_{o}\right)$ and object halfdimension $\left(\Delta x_{o}\right)$ scaled w.r.t. the Fresnel radius $r_{1}(\mathrm{~b})$.

excess attenuation $\sigma_{\ell}<5 \mathrm{~dB}$; ii) the partially obstructed (for $\left.\Delta x_{o} \simeq y_{o}\right)$ case with attenuations mostly confined in the region $5 d B<\sigma_{\ell}<10 d B$ and iii) the fully obstructed (for $\Delta x_{o}>y_{o}$ ) with $\sigma_{\ell}>10 d B$. The dashed line of Fig. 3-(b) separates the lower LOS regions from the upper NLOS ones.

\section{Stochastic MOdeling of MACHINE-TyPe CONNECTIVITY}

Industry standard applications demand for accurate prediction of the network connectivity among different machines and instruments that must be available to designers during every phase of the plant set-up. Therefore the model should be robust enough to address imperfect or inaccurate descriptions of the 3D model layout (e.g., accounting for mismatches between the simulated 3D model and the actual size, position and configuration of the field equipment). Similarly, small positioning errors of wireless instruments with respect to the simulated deployment should be also accounted for during network performance validation and stress-testing. This motivates the adoption of a stochastic model for the description of the LQI $g_{\ell}(d)(1)$ as being instrumental to link quality certification and virtual deployment. Focus of this section is on the diffraction loss $\sigma_{\ell}$ (3) that, as highlighted in Sect. 2.B, is highly sensitive to varying configurations of the obstructions. The impact on loss $g_{0}$ of imperfect positioning (2) is less relevant as confirmed by experiments (Sect. 3.B).

The proposed approach is based on the classification of the propagation into a set of $C$ mutually exclusive link types $\left\{\mathcal{C}_{1}, \mathcal{C}_{2}, \ldots, \mathcal{C}_{C}\right\}$ where each category describes a specific configuration of the building blockage and maps to a "typespecific" probability loss function $\operatorname{Pr}\left(\sigma_{\ell} \mid \ell \in \mathcal{C}_{j}\right)$. Any link 
$\ell \in \mathcal{C}_{j}$ classified as type $j$ is characterized by an excess attenuation

$$
\left[\sigma_{\ell}\right]_{d B}=\bar{\sigma}_{j}+w_{j}
$$

modeled as a log-normal random variable with $\bar{\sigma}_{j}$ being a constant term measuring the reference loss (in $\mathrm{dB}$ scale) for type $\mathcal{C}_{j}$ and $w_{j} \sim \mathcal{N}\left(0, \Delta \sigma_{j}^{2}\right)$ acting as superimposed zero-mean random fluctuations with standard deviation $\Delta \sigma_{j}=\operatorname{std}\left[\sigma_{\ell} \mid \ell \in\right.$ $\mathcal{C}_{j}$. Disturbance $w_{j}$ models the type-specific variations of the diffraction loss accounting for imperfect (i.e., unknown) layout and positioning.

In Sect. 3.A, the model parameters in (6) are at first evaluated numerically by solving the corresponding Fresnel integrals (4) for the obstruction configurations of each category, then the analytically derived parameters are validated by experiments in Sect 3.B. As described in Sect. 2.B, we consider a single obstructing interface located at position $\mathbf{x}_{O}$ and modeled as a rectangular knife-edge (vertical unbounded) surface with horizontal half-dimension $\Delta x_{o}$ and distance $y_{o}$ from the direct path. The reference loss $\bar{\sigma}_{j}=\mathbb{E}\left[\sigma_{\ell} \mid \ell \in \mathcal{C}_{j}\right]$ for type $\mathcal{C}_{j}$ is

$$
\bar{\sigma}_{j}=\mathbb{E}_{\left[\mathbf{x}_{r}, \mathbf{x}_{o} ; \Delta x_{o}\right] \in \mathcal{C}_{j}}\left[\left|\frac{E_{0}}{E_{1}\left(\mathbf{x}_{r}, \mathbf{x}_{o} ; \Delta x_{o}\right)}\right|^{2}\right]_{\mathrm{dB}},
$$

where $\mathbb{E}_{\left[\mathbf{x}_{r}, \mathbf{x}_{o} ; \Delta x_{o}\right] \in \mathcal{C}_{j}}\left[f\left(\mathbf{x}_{r}, \mathbf{x}_{o} ; \Delta x_{o}\right)\right]_{d B}$ denotes the average of $[f(\cdot)]_{d B}$ with respect to the sampled configurations of obstructions (namely the position of the object $\mathbf{x}_{O}$, the receiver $\mathbf{x}_{r}$ and the obstruction lateral half-dimension $\Delta x_{o}$ ) characterizing the link type as $\left[\mathbf{x}_{r}, \mathbf{x}_{o} ; \Delta x_{o}\right] \in \mathcal{C}_{j}$. The term $\bar{\sigma}_{j}$ can be reasonably adopted as the minimum mean square error (MMSE) predictor $\left.\hat{\sigma}_{\ell}\right|_{d B}=\bar{\sigma}_{j}$ for the nominal excess attenuation characterizing class $\mathcal{C}_{j}$. The standard deviation term with corresponding definition $\Delta \sigma_{j}=$ $\operatorname{std}_{\left[\mathbf{x}_{r}, \mathbf{x}_{o} ; \Delta x_{o}\right] \in \mathcal{C}_{j}}\left[\left|E_{0} / E_{1}\left(\mathbf{x}_{r}, \mathbf{x}_{o} ; \Delta x_{o}\right)\right|^{2}\right]_{d B}$ describes the expected fluctuations of diffraction loss around the reference $\bar{\sigma}_{j}$ for class $\mathcal{C}_{j}$ and thus certifies the criticality level of type $\mathcal{C}_{j}$ due to imperfect network layout.

\section{A. LOS and non-LOS Link Types}

From the analysis of the regions shown in Fig. 3, the proposed link types prove to fall into two macro-categories: LOS type links (the direct path and the first Fresnel region are partially unobstructed) and non-LOS (NLOS) type links (the same region is now fully obstructed). The stochastic parameters characterizing each link type (namely, the reference attenuation $\bar{\sigma}_{j}(7)$, standard deviation $\Delta \sigma_{j}$ and expected range) are evaluated numerically for sample cases of all types and summarized in Table I.

In Fig. 4 and 5, illustrative examples of typical LOS and NLOS type links are provided by highlighting a reference obstruction scenario for each case, respectively. The corresponding histograms and probability density functions (pdfs) $\operatorname{Pr}\left(\sigma_{\ell} \mid \ell \in \mathcal{C}_{j}\right)$ for sample obstruction configurations are also reported in the same Figs (on the right). The histograms (solid lines) are computed by numerical integration of Fresnel integrals (4) for each type and obtained by: i) altering the nominal position of the RX node $\mathbf{x}_{r}$ over a uniform grid

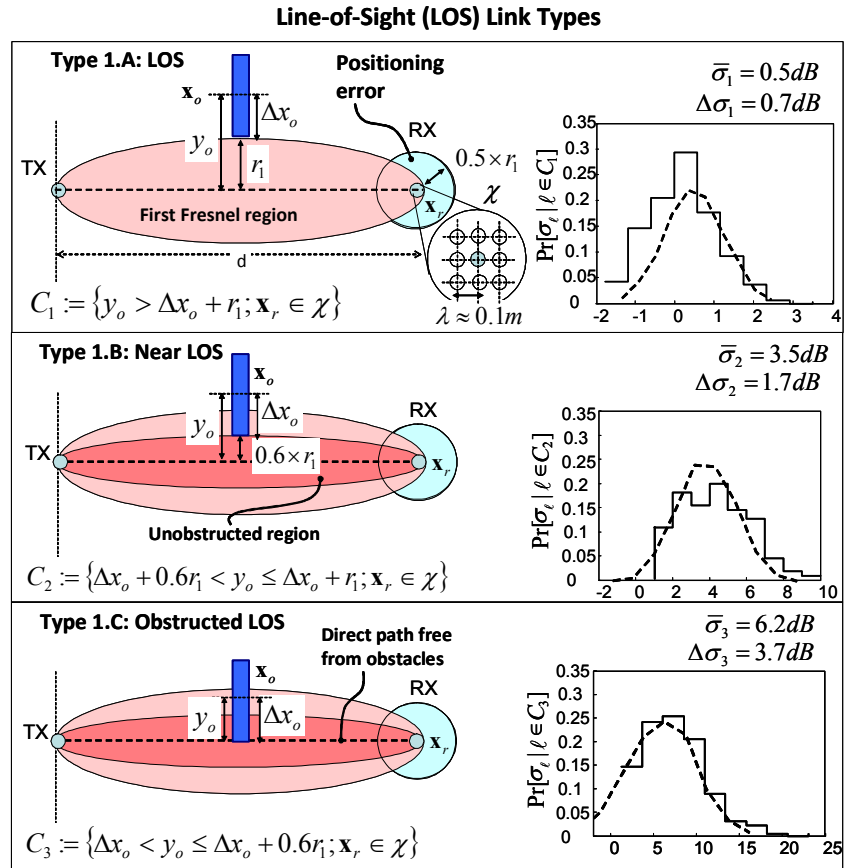

Fig. 4. From left to right. LOS link types $\mathcal{C}_{1}, \mathcal{C}_{2}, \mathcal{C}_{3}$ for rectangular vertical unbounded obstruction. Corresponding probabilities $\operatorname{Pr}\left[\sigma_{\ell} \mid \ell \in \mathcal{C}_{j}\right]$ for varying $\mathbf{x}_{r}, \mathbf{x}_{o}, \Delta x_{o}$ uniformly within the range (solid line) and superimposed log-normal model (6) (dashed lines).

\begin{tabular}{||c|c|c|c||}
\hline \hline \multicolumn{4}{|c||}{ Type 1 links (LOS) } \\
\hline \hline & $\mathrm{E}\left[\sigma \mid \ell \in C_{j}\right]$ & std $\left[\sigma \mid \ell \in C_{j}\right]$ & Max. Range $r_{j}$ \\
\hline Type 1.A $\left(C_{1}\right)$ & $\bar{\sigma}_{1}=0.5 d B$ & $\Delta \sigma_{1}=0.7 d B$ & $r_{1}=150 \mathrm{~m}$ \\
\hline Type 1.B $\left(C_{2}\right)$ & $\bar{\sigma}_{2}=3.5 d B$ & $\Delta \sigma_{2}=1.7 d B$ & $r_{2}=108 \mathrm{~m}$ \\
\hline Type 1.C $\left(C_{3}\right)$ & $\bar{\sigma}_{3}=6.2 d B$ & $\Delta \sigma_{3}=3.7 d B$ & $r_{3}=60 \mathrm{~m}$ \\
\hline \hline \multicolumn{4}{|c||}{ Type 2 links (NLOS) } \\
\hline \hline Type 2.A $\left(C_{4}\right)$ & $\bar{\sigma}_{4}=13.5 d B$ & $\Delta \sigma_{4}=5.7 d B$ & $r_{4}=32 \mathrm{~m}$ \\
\hline Type 2.B $\left(C_{5}\right)$ & $\bar{\sigma}_{5}=21 d B$ & $\Delta \sigma_{5}=5.8 d B$ & $r_{5}=15 \mathrm{~m}$ \\
\hline \hline
\end{tabular}

TABLE I

PARAMETERS FOR (TYPE 1) AND (TYPE 2) MACHINE-TYPE LINKS.

$\mathbf{x}_{r} \in \mathcal{X}$ of spacing $0.1 m$ and size $0.5 r_{1}$ for all the scenarios; ii) varying the configuration of obstruction considering a reference rectangular knife-edge surface and accounting for imperfect obstruction layouts (i.e., by uniformly varying the horizontal half-dimension $\Delta x_{o}$ and the distance $y_{o}$ from the LOS according to the chosen link type). Log-normal pdfs having the same parameters $\bar{\sigma}_{\ell}$ and $\Delta \sigma_{\ell}$ of the links $\ell \in \mathcal{C}_{j}$ (6) (dashed lines) are also depicted for direct comparison.

LOS machine-type links are characterized by objects that partially obstruct the 1st Fresnel region (and the direct path). The links are described by the following classes.

Type 1.A: $\operatorname{LOS}\left(\mathcal{C}_{1}\right)$ link-type is characterized by the lack of obstacles (with dimensions larger than the signal wavelength $\lambda$ ) within the 1st (and 2nd) Fresnel volume, while obstacles could instead occupy the remaining volumes. Considering the reference rectangular knife-edge surface example in Fig. 4, the obstruction configurations corresponding to Type 1.A links are thus characterized by $\mathcal{C}_{1}:=\left\{y_{o}>\Delta x_{o}+r_{1} ; \mathbf{x}_{r} \in \mathcal{X}\right\}$.

Type 1.B: near-LOS $\left(\mathcal{C}_{2}\right)$ link-type is observed in environ- 


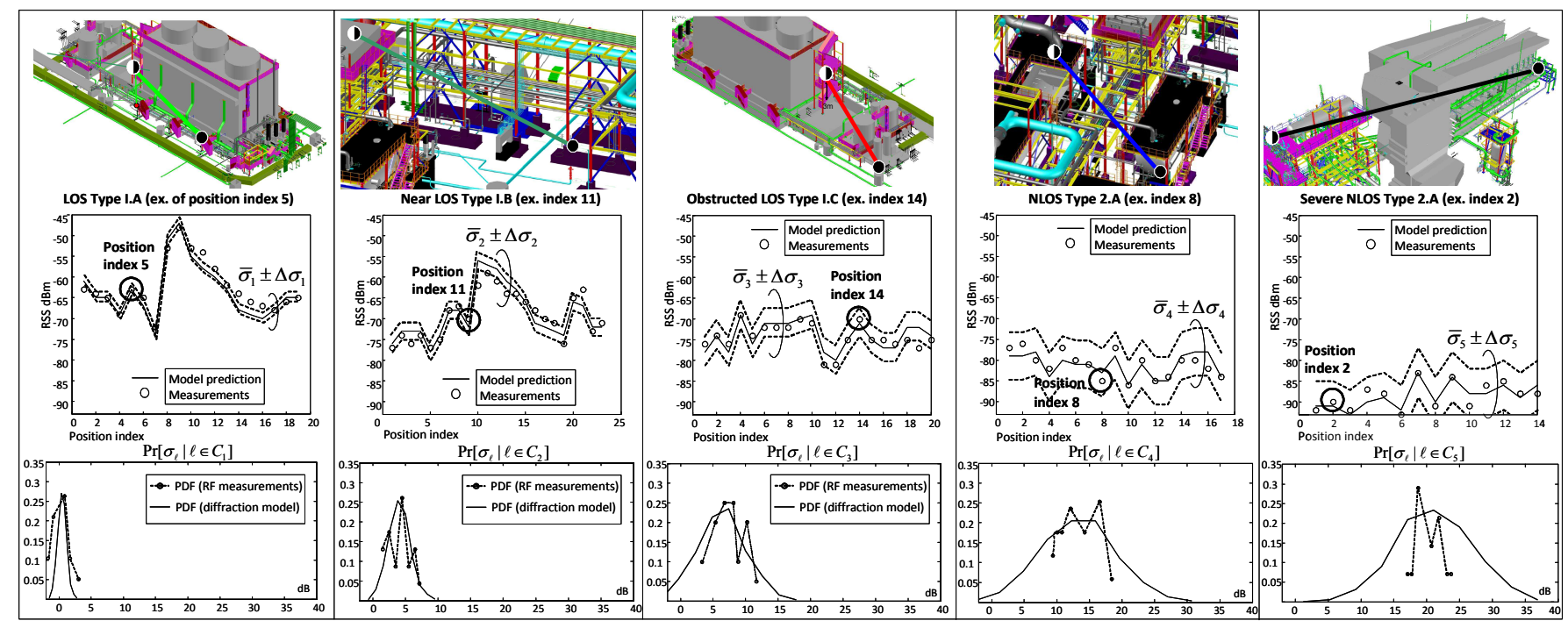

Fig. 6. Validation of machine-type stochastic connectivity model with experimental data (dots) in oil refinery sites over two interference-free IEEE 802.15 .4 2.4GHz channels (15 and 23). Examples of deployment testing from the 3D CAD (ref. by position index) are illustrated for each class (top-figures).

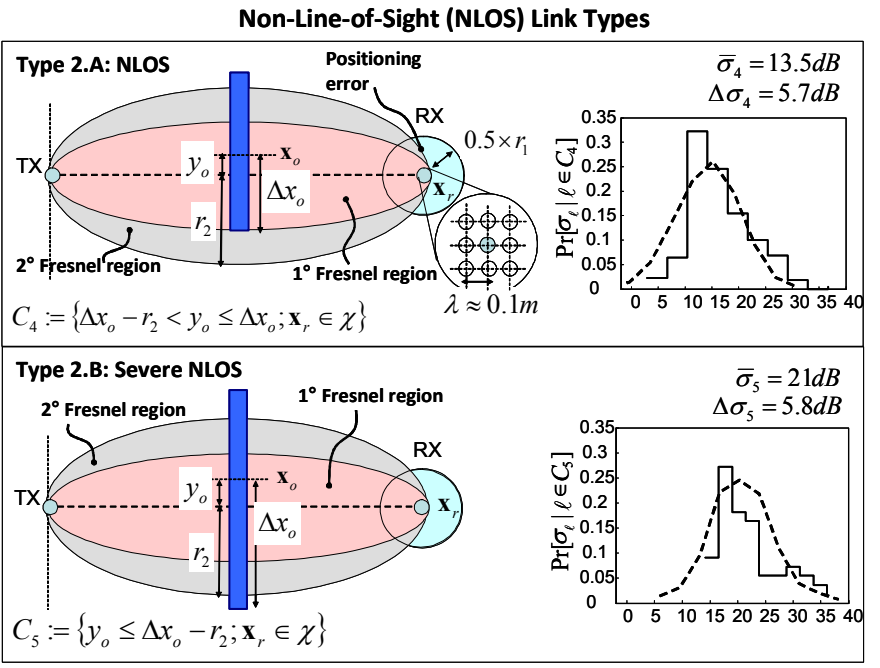

Fig. 5. NLOS link types $\mathcal{C}_{4}, \mathcal{C}_{5}$ with corresponding parameters as in Fig. 3.

ments where the obstacles are located in the 1 st Fresnel outer region at distance $0.6 \times r_{1}$ from the direct path. This 'forbidden' region is used here as a criterion to decide whether an object is to be treated as a relevant obstruction. Considering the reference example in Fig. 4, Type 1.B links are characterized by $\mathcal{C}_{2}:=\left\{\Delta x_{o}+0.6 r_{1}<y_{o} \leq \Delta x_{o}+r_{1} ; \mathbf{x}_{r} \in \mathcal{X}\right\}$.

Type 1.C: obstructed-LOS $\left(\mathcal{C}_{3}\right)$ link-type is observed in environments where the obstacles are located inside the forbidden region, although the direct path connecting the transmitter and the receiver is still unobstructed. The reference obstruction configurations are thus characterized by $\mathcal{C}_{3}:=\left\{\Delta x_{o}<y_{o} \leq \Delta x_{o}+0.6 r_{1} ; \mathbf{x}_{r} \in \mathcal{X}\right\}$. Compared to LOS types 1.A and 1.B, model inaccuracies might cause the diffraction loss to experience two-times larger deviations from the corresponding reference value of $\bar{\sigma}_{3}=6.2 \mathrm{~dB}$ as $\Delta \sigma_{3}=3.7 d B$.
NLOS machine-type links are characterized by objects now obstructing the direct path. For all cases, the analysis highlights possibly larger fluctuations $w_{j}$ (6) from the observed reference losses $\bar{\sigma}_{j}$, compared to LOS types, with standard deviation now $\Delta \sigma_{j}=5 \div 6 \mathrm{~dB}$. The following link types are therefore deployment-critical and should be carefully addressed during network layout design (see Sect. 5-6).

Type 2 A: NLOS $\left(\mathcal{C}_{4}\right)$ link-type is characterized by objects obstructing the 1st Fresnel region, but leaving the remaining higher-order Fresnel regions mostly free from obstacles. As illustrated in Fig. 5, the corresponding configurations of the obstruction are $\mathcal{C}_{4}:=\left\{\Delta x_{o}-r_{2}<y_{o} \leq \Delta x_{o} ; \mathbf{x}_{r} \in \mathcal{X}\right\}$, where $r_{2}=r_{1} \sqrt{2}$ is the radius of the 2 nd Fresnel region.

Type 2.B severe-NLOS $\left(\mathcal{C}_{5}\right)$ link-type refers to a severe NLOS environment where the 1st and the 2nd Fresnel regions are completely obstructed by one or more obstacles with significant size (and dimensions scaling as $\sim 4 \div 5 r_{1}$ ). The corresponding configurations of the obstruction are thus characterized by $\mathcal{C}_{5}:=\left\{y_{0} \leq \Delta x_{o}-r_{2} ; \mathbf{x}_{r} \in \mathcal{X}\right\}$.

\section{B. Model validation and measurements campaigns}

In this section, the proposed model is validated according to a database of radio measurements taken in different refinery sites. In the proposed set-up, we deployed absolute and gauge pressure ISA IEC62734 [8] compliant FDs communicating directly with a single GW node connected to a Fieldbus backbone network. Omnidirectional antennas are employed for both FD and GW nodes. The experiments have been carried out in three sites (see the illustrative examples in Fig. 6) within the same oil refinery [11]: the first site is a $100 \mathrm{~m} \times 200 \mathrm{~m}$ area around a flare unit, the second one is a $60 \mathrm{~m} \times 30 \mathrm{~m}$ area surrounding a furnace structure, while the last one is a $120 \mathrm{~m} \times 45 \mathrm{~m}$ cooling unit building area. All scenarios under consideration cover most of the representative link types identified in Sect. 3.A and are characterized by metallic objects and concrete buildings with high reflectivity surfaces. 
Before the tests, we used a signal analyzer to characterize the interferers in the area (in terms of received power and occupied bandwidth). In these tests no relevant activity was detected so that the contribution of interference $n_{\ell}$ (1) captured by the receiver can be neglected. Two IEEE 802.15.4 channels are selected for the experiments with center frequencies 2.405 $\mathrm{GHz}$ and $2.480 \mathrm{GHz}$, corresponding to the IEEE 802.15.4 channel numbers 15 and 23 , respectively.

We adopt the model (6) for the prediction of the LQI

$$
\left[\hat{g}_{\ell}\right]_{d B}=\left[g_{0}(d)\right]_{d B}-\bar{\sigma}_{\ell}
$$

Predictions are compared with corresponding RSS measurements $\gamma_{\ell}$ capturing also the effect of dynamic component $s_{\ell}$ and disturbance $n_{\ell}$. The parameters characterizing the distance dependent component $\left[g_{0}\right]_{d B}$ in (2), namely $\left(h_{R}, h_{T}, d\right)$, are obtained from $3 \mathrm{D}$ CAD models of the structures. The freespace channel gain $g_{0}\left(d_{0}\right)=-47 \mathrm{dBm}$ for $d_{0}=2 \mathrm{~m}$ is used to set the reference level. The measured path loss exponents for a typical case of $d_{F} \simeq 50 \mathrm{~m}\left(\mathrm{GW}\right.$ at $h_{T}=6 \mathrm{~m}$ above ground, FDs at ground level) are $\alpha_{0}=2$ and $\alpha_{F}=2.5$, respectively.

The selection of link type $\mathcal{C}_{j}$, and thus of the reference loss $\bar{\sigma}_{j}(7)$, is based on the geometrical information extracted from 3D CAD databases by considering only obstacles with size comparable to $\lambda$. The interface to the existing planning is implemented by CAD software, classification is based on inspection of the corresponding 3D environment. Before link classification, data-base simplification should be applied to automatically (or manually in some cases) remove objects not relevant as $2 \mathrm{D}$ surfaces or 3D polygons with size smaller than the wavelength $\lambda$ as practically irrelevant for propagation impact. For a selected link (identified by the coordinates of the node pair) we generate a beam of rays that uniformly span the first and second Fresnel region around the transmitter and the receiver. The spacing among rays (offset) is comparable to the wavelength $\lambda$. The obstruction area is thus identified by collecting and reporting the positions of the intersections (or clash points) between the rays and the possible 3D solid obstruction(s) located in-between the coordinates of nodes. The link type is chosen from the identified classes in Sect. 3.A as the one that best matches the observed obstruction area. Notice that in more simple LOS or OLOS settings, the number of probing rays can be reduced based on an preliminary inspection (e.g., visual) of the 3D CAD.

In Fig. 6 the measurements (empty dots) of RSS are grouped by link type and compared with the predicted LQI values (solid lines). The dashed lines indicate the confidence interval ( $\pm \Delta \sigma$ range) for each class. Prediction errors are caused by three main factors: $i$ ) modeling mismatches or link classification errors due to meaningful layout inaccuracies and mismatches between the 3D model (e.g., after database simplification) and the real environment; ii) RSS fluctuations due to electromagnetic interference ${ }^{3}$ over the $2.4 \mathrm{GHz}$ band or HW errors due to varying temperature and environmental conditions; iii) fading induced by objects or people moving (mostly temporarily) in the area [6]. The comparison highlights

\footnotetext{
${ }^{3}$ Caused by special instruments and industrial machines (e.g., induction furnaces).
}

the accuracy of the proposed approach. Prediction of LQI $\hat{g}_{\ell}(8)$ is reasonably tight for LOS types (with errors below $2 \div 3 \mathrm{~dB}$ ) while smaller accuracy (approx. $5 \mathrm{~dB}$ ) is observed for NLOS types, as expected from the log-normal model (6). In the same Fig. 6 (see corresponding figures at bottom) modeling of loss fluctuations $w_{j} \sim \mathcal{N}\left(0, \Delta \sigma_{j}^{2}\right)$ is also validated for each class. As expected, compared to LOS types, larger deviations $\Delta \sigma_{j}$ are observed for NLOS and increase with the size of the obstructed region.

Excluding measurement errors due to the RF devices themselves (i.e., accuracy in the order of +/- 1dB), RSS measurements are very stable over time while fluctuations are mainly due to environmental effects (e.g., temperature) and fading caused by moving objects or people (e.g., operators) in the network area. The optimization of network deployment introduced in Sect. 5 is performed in order to exploit spatial redundancy and thus avoiding these temporary RSS fluctuations. Of course, if these fluctuations are due to permanent plant changes (e.g., for expansions of existing sites), then a post-layout verification step is mandatory.

The previous considerations about LQI predictions apply to omnidirectional antennas. However, in the general case, the specific radiation pattern of the node antennas must be included. This implies that an additional attenuation term must be included in eq. (8). The antenna radiation pattern is an additional degree of freedom that can be exploited in the deployment phase (e.g., to attenuate spatially localized interferences).

\section{Post-layout verification}

Before the plant start up, post-layout verification should be performed to certify the deployments of nodes by acquiring a complete set of radio measurements within the plant site and by verifying each link. One or more errors found in this phase require additional nodes placement (or repositioning of existing nodes) followed by additional radio link measurements and validation steps, thus slowing down the plant activation and adding extra costs. Moreover, inaccuracies during the radio planning design phase will therefore turn into issues during the commissioning phase of the plant. To avoid or at least mitigate these problems, the proposed machine-type link model provides the ability to capture pre-deployment information about those links that could require some replanning (namely NLOS types). An example of post-layout verification is performed on a set of measurements not used for model validation and it is described in Sect. 5.B.

\section{Coexistence With WiFi Networks}

It is envisioned that most of FoT related wireless technologies will coexist over the same spectrum with other devices employing different radio protocols. This is particularly true for the popular WiFi and IEEE 802.15.4 based standards operating in the same unlicensed $2400-2490 \mathrm{MHz}$ ISM band. The possibility to exploit multiple and heterogeneous network technologies deployed in close proximity (i.e., for monitoring/controlling the same industrial process [8]) provides an attractive opportunity for efficient resource sharing 
IEEE 802.15.4 (standard 250 Kbps - DSSS $Q_{1}=8$ )

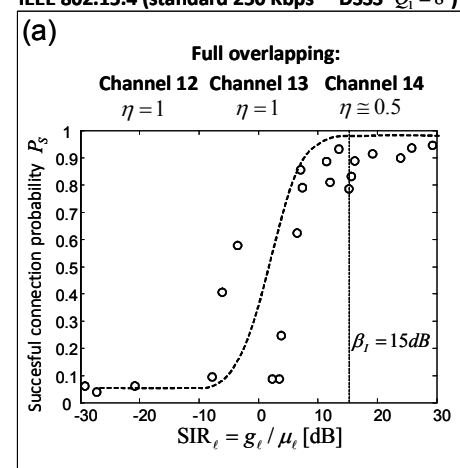

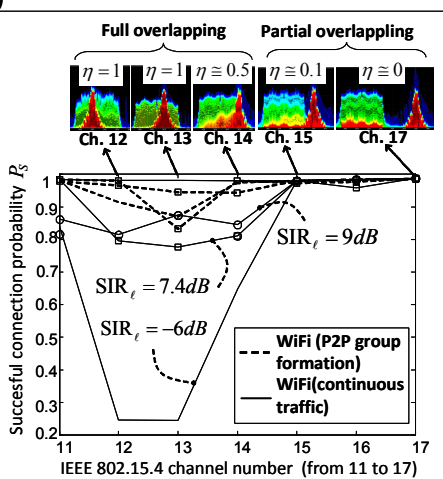

IEEE 802.15.4 PHY (enhanced PHY rate $1 \mathrm{Mbit} / \mathrm{s}$ - DSSS $Q_{2}=2$ )

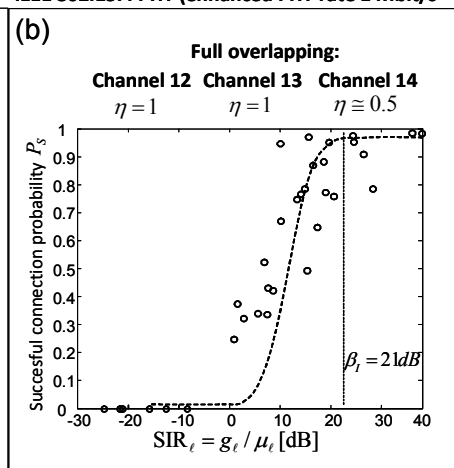

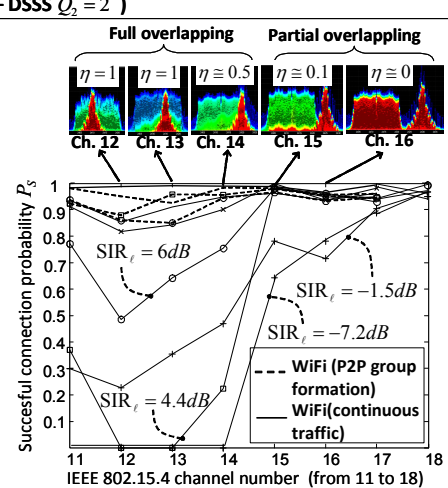

Fig. 8. (a) From left to right. Succesful probablity for varying SIR under full overlapping (left). Succesful probability for varying WiFi - IEEE 802.15 .4 overlapping (right), for selected values of SIR and traffic loads, under continuous transmission and P2P WiFi group formation; (b) Same results of (a) now with enhanced IEEE 802.15.4 PHY data-rate of $1 \mathrm{Mbit} / \mathrm{s}$ and reduced spreading factor to 2 .

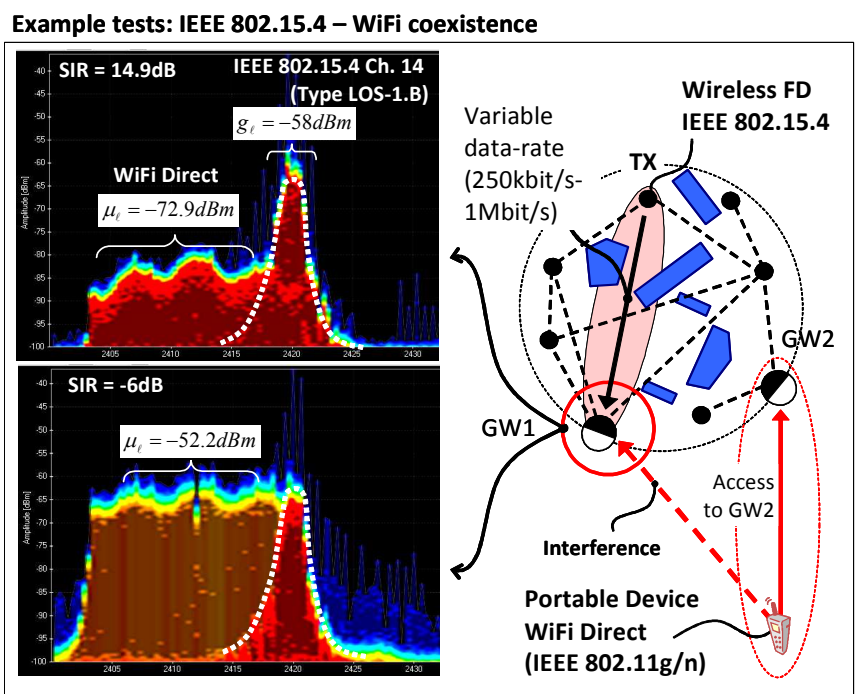

Fig. 7. Heterogeneous WiFi-IEEE 802.15.4 network scenario: a portable WiFi-enabled device is communicating with GW2 by WiFi Direct. Interference (with varying power $\mu_{\ell}$ and SIR, see two examples) is measured at GW1 by the spectrum analyzer. The IEEE $802.15 .4 \mathrm{GW} 1$ receiver is thus suffering as victim of WiFi interference.

and traffic off-loading. However, the practical deployment of heterogeneous radio technologies still needs to face some critical issues and design challenges such as the development of an efficient regulatory framework that preserves Quality of Service (QoS) by interference coordination and network design mechanisms to ensure that the mutual interference is kept below acceptable limits.

So far we illustrated a model for prediction of LQI $g_{\ell}$. However, the LQI metric is not enough to estimate the average successful connection probability $P_{S}$ in the presence of interference [18]. Fig. 7 illustrates the problem of coexistence of $2.4 \mathrm{GHz}$ machine-type communications conforming to IEEE 802.15.4 and WiFi standards. In particular, we focus on the relevant case (in terms of QoS [22]) of IEEE 802.15.4 devices suffering as victims of one WiFi mobile devices sending data to a GW acting as a cloud access node. The traffic load of the interference $\mu_{\ell}$ is modeled as Bernoulli process with probability $0 \leq \mathrm{P}_{\mu_{\ell}} \leq 1$ accounting for the degree of frame collisions. In what follows, the successful probability $P_{S}$ is defined as a function of pre-deployment information. It thus accounts for layout inaccuracies where the obstruction loss $\sigma_{\ell}$ is modeled as lognormal random variable as shown in Sect. 3 ; in addition it also considers the interference originated by WiFi with power $\mu_{\ell}$ and probability $\mathrm{P}_{\mu_{\ell}}$. For any link $\ell \in C_{j}$, the Signal to Interference Ratio $\mathrm{SIR}_{\ell}=g_{\ell} / \mu_{\ell}$ serves as an additional metric to LQI for successful connection probability $P_{S}=P_{S}\left(\mathcal{C}_{j}\right)$ assessment:

$$
P_{S}=\left\{\begin{array}{c}
\operatorname{Pr}\left[g_{\ell}>\beta\right]=\Psi\left(\beta \mid \mathcal{C}_{j}\right), \text { if } \mu_{\ell}<\frac{\beta}{\beta_{I}} \\
\mathrm{P}_{\mu_{\ell}} \operatorname{Pr}\left[\operatorname{SIR}_{\ell}>\beta_{I}\right]+\left(1-\mathrm{P}_{\mu_{\ell}}\right) \operatorname{Pr}\left[g_{\ell}>\beta\right], \text { if } \mu_{\ell} \geq \frac{\beta}{\beta_{I}}
\end{array}\right.
$$

where model for LQI $g_{\ell}$ is based on (1) with $\sigma_{\ell}$ in (6), while

$$
\Psi\left(x \mid \mathcal{C}_{j}\right)=\operatorname{erf}\left[\frac{\left[x^{-1} g_{0}(d)\right]_{d B}-\bar{\sigma}_{j}}{\Delta \sigma_{j}}\right]
$$

depends on link type $\mathcal{C}_{j}$ and erf [.] is the error function. Similarly, the probability term is defined as $\operatorname{Pr}\left[\operatorname{SIR}_{\ell}>\beta_{I}\right]=$ $\Psi\left(\beta_{I} \mu_{\ell} \mid \mathcal{C}_{j}\right)$ with $\Psi\left(x \mid \mathcal{C}_{j}\right)$ in (10). The ratio $\beta / \beta_{I}$ indicates the critical value of co-channel disturbance $\mu_{\ell}$ captured by the receiver above which interference (SIR and collision probability $\mathrm{P}_{\mu_{\ell}}$ ) has a relevant impact on connectivity. The RSS threshold $\beta=-85 \mathrm{dBm}$ [15] depends on receiver sensitivity and limits the performance in interference-free scenarios. Sensitivity $\beta$ also depends on IEEE 802.15.4 PHY data-rate settings as described in Sect. 4.

As experimentally verified in Sect. 4.A, the threshold $\beta_{I}=$ $\beta_{I}(\eta)$ (or link margin) critically depends on the degree of spectrum overlapping $\eta \in[0,1]$ between the useful signal and the co-channel WiFi disturbance. Overlapping is defined in terms of the amount of interference power as $\eta \times \mu_{\ell}$ lying over the considered IEEE 802.15.4 channel: this is obtained by considering only the portion of WiFi spectrum in common with the IEEE 802.15.4 signal. In what follows, the threshold $\beta_{I}(\eta)$ is evaluated experimentally for the relevant case (in the industrial context) of IEEE 802.15.4 devices acting as victims of WiFi IEEE $802.11 \mathrm{~g}$ interference and subject to full $(\eta \geq 0.5)$ or partial $(\eta<0.5)$ spectrum overlapping. In 
particular, the settings consider critical (or worst-case) high traffic load scenarios where both WiFi and IEEE 802.15.4 networks are possibly continuously transmitting. i.e. to comply with safety-critical industry-standard applications. Other interference coexistence scenarios are considered and analyzed in [18]-[19]. Below, the impact of enhanced IEEE 802.15.4 PHY data-rate transmission mode is also discussed. The topic is relevant as enhanced data-rate mode is supported by recent HW designs [20] to comply with delay-sensitive applications.

\section{A. IEEE 802.15.4 and WiFi coexistence for high traffic loads}

The coexistence among IEEE 802.15.4 devices sharing the spectrum with WiFi-enabled devices is analyzed by experiments, in accordance with a set of pre-defined conditions on LQI, spectrum overlapping and WiFi traffic load responsible for frame collisions. The set-up is depicted in Fig. 7. It consists of one IEEE 802.15.4 device that transmits full data frames of 127 bytes towards a GW labeled as node 1 (GW1). GW nodes support double radio technology with WiFi and IEEE 802.15.4. The transmitter is a programmable device configured to switch among 7 consecutive channels having bandwidth $5 \mathrm{MHz}$ (with center frequencies [18] ranging from $2405 \mathrm{MHz}$ to $2435 \mathrm{MHz}$ ). It sends data in continuous mode ${ }^{4}$ by disabling carrier sense multiple access (CSMA) to conform with industry standard PHY [8] and implement a directsequence spread spectrum (DSSS) with factor $\left(Q_{1}=8\right)$, and data-rate of $250 \mathrm{kbps}$. The $\mathrm{GW}$ receiver 1 might be affected by a disturbance (co-channel interference) originated by a $\mathrm{WiFi}$ enabled portable Android device communicating in peer-topeer (P2P) mode with GW node 2 (GW2) through WiFi-Direct [23] (over IEEE 802.11g) using the band $2400-2420 \mathrm{MHz}$. These interference scenarios are characterized by varying powers $\mu_{\ell}$, collision probability $\mathrm{P}_{\mu_{\ell}}$ and spectrum overlapping $\eta$, both measured by a $2.4 \mathrm{GHz}$ spectrum analyzer.

In Fig.8-(a) we analyze the successful connection probability $P_{S}$ for varying SIR (9) assuming full overlapping $\eta \geq 0.5$ (channels $11-14$ ) and continuous WiFi traffic, with high load as $\mathrm{P}_{\mu_{\ell}} \simeq 1$. Successful probability is obtained by counting the number of successfully acknowledged data frames normalized by the number of frames received with interferer disabled. According to model (9), the optimal threshold $\beta_{I}$ can be reasonably set to $\beta_{I}=15 d B$. As confirmed from (11), the use of channels experiencing $\eta \geq 0.5$ must be avoided by blacklisting (when possible) for $\mathrm{SIR}_{\ell}<15 d B$. In the same Fig. 7-(a), probability $P_{S}$ is also evaluated over 7 consecutive channels to highlight the impact of spectrum overlapping and interference traffic loads. The analysis focuses on the extreme cases of full overlapping with $\eta \simeq 1$ (channels $11-13$ ) and $\eta \simeq 0.5$ (channel 14), and partial overlapping with $\eta<0.1$ (channels $15-17$ ), being the most meaningful cases observed in the tests. For partial overlapping, disturbance is now due to out-of-band spurious emissions of WiFi devices. We also consider WiFi Direct P2P group formation [23] (in dashed lines), with collision probability $\mathrm{P}_{\mu_{\ell}} \simeq 0.1$ and continuous WiFi traffic (in solid lines), with $\mathrm{P}_{\mu_{\ell}} \simeq 1$. The use of partially overlapped channels $(15-17)$ might be tolerated

\footnotetext{
${ }^{4}$ with ack. and beacon frame options enabled (beacon order 4)
}

without significant penalties even at low SIR regime (when $\left.\mathrm{SIR}_{\ell}>-6 d B\right)$. A reasonable approximation to threshold values (in $\mathrm{dB}$ scale) for SIR in (9) is found as

$$
\beta_{I}\left(\eta, Q_{1}=8\right) \simeq\left\{\begin{array}{cl}
15 d B & \text { for } \eta \geq 0.5(\text { ch. } 11-14) \\
-6 d B & \text { for } \eta<0.5(\text { ch. } 15-17)
\end{array},\right.
$$

these can be used for connectivity prediction (see Sect. 6).

\section{B. Enhanced IEEE 802.15.4 PHY data-rate}

In this section we investigate the use of high-data rate mode as available in recent low-power IEEE 802.15.4 compliant transceiver devices [20]. The use of the enhanced data-rate mode can be a promising option for fast servicing of unexpected conditions that require a fast reaction over the network in a low-latency mode and a meaningful increase of the sensor data publishing rate [21]. In Fig. 8-(b), the coexistence with WiFi is addressed for the same settings, now by programming the FDs to reduce the IEEE 802.15.4 DSSS factor (for payload transmission) down to a value of $Q_{2}=2$, corresponding to a PHY data rate of $1 M b p s$. According to our experiments, the IEEE 802.15.4 data frame transmission duration reduces from $4 \mathrm{~ms}$ down to $1.6 \mathrm{~ms}$ (for a payload of 102 bytes), at the cost of a slightly lower interference-free sensitivity $\beta \simeq-82 \mathrm{dBm}$, compared to the standard data-rate case. Given that the IEEE 802.15.4 transceiver is continuously transmitting, the observed collision probability $\mathrm{P}_{\mu_{\ell}}$ during $\mathrm{P} 2 \mathrm{P}$ WiFi group formation is marginally influenced by the reduced transmission duration (and still $\mathrm{P}_{\mu_{\ell}} \simeq 0.1$ ). The optimal SIR threshold is now $\beta_{I}=\beta_{I}\left(\eta, Q_{2}=2\right) \simeq 21 \mathrm{~dB}$, and therefore can be reasonably modeled as a linear function of (11)

$$
\beta_{I}\left(\eta, Q_{2}=2\right) \simeq \beta_{I}\left(\eta, Q_{1}\right)+\left[Q_{1} / Q_{2}\right]_{d B},
$$

as increasing with the ratio of spreading factors $\left[Q_{1} / Q_{2}\right]_{d B}=$ $6 d B$ to account for the larger interference capturing effect.

\section{GRAPH THEORETIC DESIGN OF CLOUD-BASED SENSOR NETWORKS}

The simulation and layout design tool supporting cloud connectivity optimization is fairly general and applicable in different industrial scenarios. The tool assigns the nominal positions of nodes and link types obtained from 3D CAD analysis. A virtual representation of the deployed instrument is obtained by modeling the layout inaccuracies and imperfect positioning responsible for log-normal deviations of the obstruction loss from the reference term $\bar{\sigma}_{j}(6)$.

Graph theory is the natural framework for the exact mathematical modeling of complex network structures [12] characterized by dense interconnected objects. In what follows, the industrial network is modeled by an undirected geometrical graph $\mathcal{G}(\mathcal{N}, \mathcal{V})$ consisting of a set $\mathcal{N}=\{1,2, \ldots N\}$ of $N$ vertices identifying the position of the FDs and of the GWs deployed in an arbitrary industrial site. The elements of $\mathcal{V}$ are the links $\ell$ connecting pairs of elements in $\mathcal{N}$. FDs connect to each other probabilistically, depending on their link type: connectivity for link $\ell$ among two devices $(a, b) \in \mathcal{N}$ is simulated by: $i$ ) assigning a class $\ell \in \mathcal{C}_{j}$ and the obstruction loss $\sigma_{\ell}$ in (6) according to the corresponding 3D model of 
the plant (see Sect. 3); ii) configuring the binary symmetric adjacency matrix [12] $\mathbf{C}(\mathcal{G})$ with $[\mathbf{C}(\mathcal{G})]_{a, b}=c_{a, b}$, null elements along the main diagonal and $\operatorname{Pr}\left[c_{a, b}=1\right]=P_{S}$. Probability $P_{S}=P_{S}\left(\mathcal{C}_{j}\right)$ depends on class $\mathcal{C}_{j}$ and it is given by model (9).

The proposed network layout optimization consists of four phases:

Network structure identification. The modular structure of the network is analyzed to identify potential weaknesses of the layout. A spectral graph partitioning method [12] is used to identify possibly disconnected or weakly connected network structures over the deployment $\mathcal{G}$. Weakly connected networks are defined in general as groups (or clusters) of wireless devices such that there is a higher density of links within groups than between them. A cluster of $\mathcal{G}$ is thus a subset of $\mathcal{N}$ that is richly intra-connected (cohesive group) but sparsely connected with the remaining vertices of the graph. Weakly connected groups can be considered as the bottleneck of the cloud system and potentially critical in case of layout inaccuracies or interference. The cohesive groups can be identified by analyzing the eigenvalues and eigenvector pairs $\left(\lambda_{i}, \mathbf{v}_{i}\right)$ of the Laplacian matrix [24]

$$
\mathbf{L}(\mathcal{G})=\mathbf{K}(\mathcal{G})-\mathbf{C}(\mathcal{G})
$$

of graph $\mathcal{G}$ where $\mathbf{K}(\mathcal{G})=\operatorname{diag}\left(k_{1}, \ldots, k_{a}, \ldots, k_{N}\right)$ and

$$
k_{a}=\sum_{b=1}^{N} c_{a, b}
$$

is the degree of the device $a$. Being for Laplacian matrices $\lambda_{1}=0$, the $N$ length eigenvector components $\mathbf{v}_{2}, \ldots, \mathbf{v}_{Q+1}$ corresponding to the $Q$ consecutive Laplacian eigenvalues $\lambda_{2}, \ldots, \lambda_{Q+1}$ lying in a small gap away from zero provide a virtual representation of $Q+1$ weakly connected FDs sub-networks characterized by dense connectivity within each group [24]. Graph partitioning can be improved using several refinement techniques [25]. An effective method is to look for the links in the network that are responsible for connecting many pairs of devices (e.g., by shortest path) and assigning them an high cost metric ("betweenness" [26]). Network components emerge by removing progressively the links with the largest betweenness: these links are thus critical for relay deployment.

Relay assignment for topology control. The physical structure of the network in terms of node adjacencies is designed for connectivity and topology control. The proposed deployment algorithm iteratively adds new relay nodes among $M$ candidate positions (namely identified during pre-commissioning of the plant) to guarantee robust connectivity, therefore by increasing the number of edges running between weakly connected clusters (namely the cut size). The method can be configured to implement several metrics for optimal relay placement (see [27] for a survey). Based on the model of Sect. 3, a reasonable strategy adopted here is to deploy new relays $i$ ) to strengthen connectivity of weakly connected groups using the algebraic connectivity [28] as performance metric (see the following section) and ii) to minimize the use of critical NLOS type links (Type 2), being highly sensitive to 3D model inaccuracies and interference. When $R \leq M$ relay nodes are added, they form a new "augmented" graph $\mathcal{G}^{(R)}$ while the relay placement stops when the algebraic connectivity, corresponding to graph $\mathcal{G}^{(R)}$ satisfies a target value $\xi$.

Interference stress-testing. The connectivity performance is finally tested under a simulated WiFi shared access scenario. Stress-testing of connectivity is thus implemented to determine the safe usage limits of the chosen layout in interferencelimited environments characterized by the tuple $\left(\mathrm{P}_{\mu_{\ell}}, \mu_{\ell}, \eta\right)$ according to (9).

Post-layout verification. Finally, the node set that implements the proposed connectivity graph is deployed in the real plant and its performance is verified by measurements. Although not explicitly addressed in this paper, post-layout verification is an iterative step where connectivity errors, if found, are identified and the initial nodes deployment is modified focusing on the sub-graphs that do not fulfill the post-deployment testing. As illustrated in the case study of Sect. 5.B, the layout verification confirms the effectiveness of the predicted deployment without the need of additional iterations. In general, the accuracy of the model (see Sect. 3.B) is enough to provide a meaningful reduction of the iterations needed during the on-site network set-up.

\section{A. Performance metrics}

Different approaches to relay deployment for topology control will produce different network designs: it is therefore useful to provide key metrics to assess the usefulness of a network structure as the output of the chosen optimization parameters.

Algebraic connectivity. Algebraic connectivity [28] provides a powerful metric to assess the quality of information flow inside the cloud network and the connectivity robustness. The algebraic connectivity $\lambda_{2}$ is defined as the second smallest eigenvalue of the Laplacian (13) $\mathbf{L}(\mathcal{G})$ of graph $\mathcal{G}$. Let the information load exchanged by any cloud node pair $(a, b)$ at time $t$ be $x_{a}(t)$ and $x_{b}(t)$, respectively. Assuming that the information flow inside the cloud is modeled by linear consensus [29]

$$
\frac{d x_{a}(t)}{d t}=\sum_{b \neq a} c_{a, b}\left[x_{b}(t)-x_{a}(t)\right]
$$

then the connectivity $c_{a, b}$ acts as a weighting factor for the information exchange between node $a$ and $b$. For large consensus time, the solution of eq. (15) is ruled by algebraic connectivity as $x_{a}(t) \sim \bar{x}+\beta \exp \left(-\lambda_{2} t\right) \forall a$, where $\bar{x}$ is the consensus (average) state and $\lambda_{2}$ the algebraic connectivity. Therefore, the bigger $\lambda_{2}$, the faster $x(t)$ diffuses to its consensus state. In this sense, it is reasonable to optimize the network structure by defining a relay placement stopping criteria ruled by algebraic connectivity. On every new deployed relay, the algebraic connectivity of the relay-augmented graph $\mathcal{G}^{(R)}$ is computed as $\lambda_{2}\left[\mathcal{G}^{(R)}\right]$ while the relay placement stops when $\lambda_{2}>\xi$, with $\xi$ indicating a critical convergence time of the information flow inside the network. The above condition also ensures that the network connectivity can tolerate up to a maximum of $\xi$ random node (or link) failures due to model layout or machine positioning inaccuracies (and interference). In the Sect. 6, the effect of different choices of the design 
parameter $\xi$ on cloud network performance will be discussed by extensive simulations under virtual layout inaccuracies and interference.

Energy efficiency. For a variety of FoT entities, minimizing the energy to be spent for communication/computing purposes is a primary constraint [30]. It is therefore mandatory to develop solutions to optimize energy usage (even at the expenses of performance loss). Given that in recent microcontroller designs the electric charge spent for transmission is comparable to the cost for receiving [20], then the node degree $k_{a}$ (14) provides a reasonable indicator of node lifetime by counting the number of links monitored by the device $a \in \mathcal{N}$ (for transmission/reception) during each cloud activity cycle ${ }^{5}$. The electric charge subtracted by node $a$ to the battery (energy cost) during one activity cycle is thus approximated as

$$
E_{a}=k_{a} \times E_{T-R}+E_{S}, \forall a \in \mathcal{N}
$$

where $E_{T-R}$ accounts for the electric charge spent by the transceiver in active state while $E_{S}$ is the cost of active internal oscillator and RAM (e.g., sleep mode). Node lifetime can be modelled as $T_{\text {life }}=T_{\text {cycle }} \times C / E_{a}$ where $T_{\text {cycle }}$ measure the duration of one cloud activity cycle and $C$ is the battery capacity.

Data-rate and QoS. In machine-type communications there are several scenarios where a somewhat large amount of delay-tolerant data needs to be transferred, with a temporary larger data rate. Typical application cases include over-theair programming of devices deployed on field or acquisition of logging-information. The use of low-power IEEE 802.15.4 devices with improved (and run-time programmable) PHY data-rates can be therefore crucial to support these unconventional traffic patterns. Impact of reduced range and higher interference capturing effects (Sect. 4) should be properly accounted for during layout stress-testing.

Delay sensitivity. To comply with delay sensitive industrial applications, network layouts should be designed so that any path from GWs to FDs, and among any cloud device pair, experiences small enough geodesic distance (in terms of number of hops). This is typically required for fast servicing of asynchronously generated and "sporadic" events or during the management of safety-critical conditions. The average shortest path metric $\Gamma$

$$
\Gamma=\frac{1}{(N+R)(N+R-1)} \sum_{(a, b) \in \mathcal{N}, a \neq b} d_{a, b},
$$

is defined as the mean of the geodesic length $d_{a, b}$ over all the node pairs of the augmented graph $\mathcal{G}^{(R)}$ (of $N+R$ nodes) and it is a convenient metric for performance assessment.

\section{B. Case study in an oil refinery site}

The case study of Fig. 9 shows a practical example of layout design for industrial sensor network. The wireless instruments (gauge pressure sensors) are deployed in a $120 \mathrm{~m} \times 45 \mathrm{~m}$ oil refinery site where a cooling tower unit causes the network

\footnotetext{
${ }^{5}$ The activity cycle might correspond to one superframe (e.g., of duration $1 s$ for IEC 62591 and also typ. for IEC 62734).
}
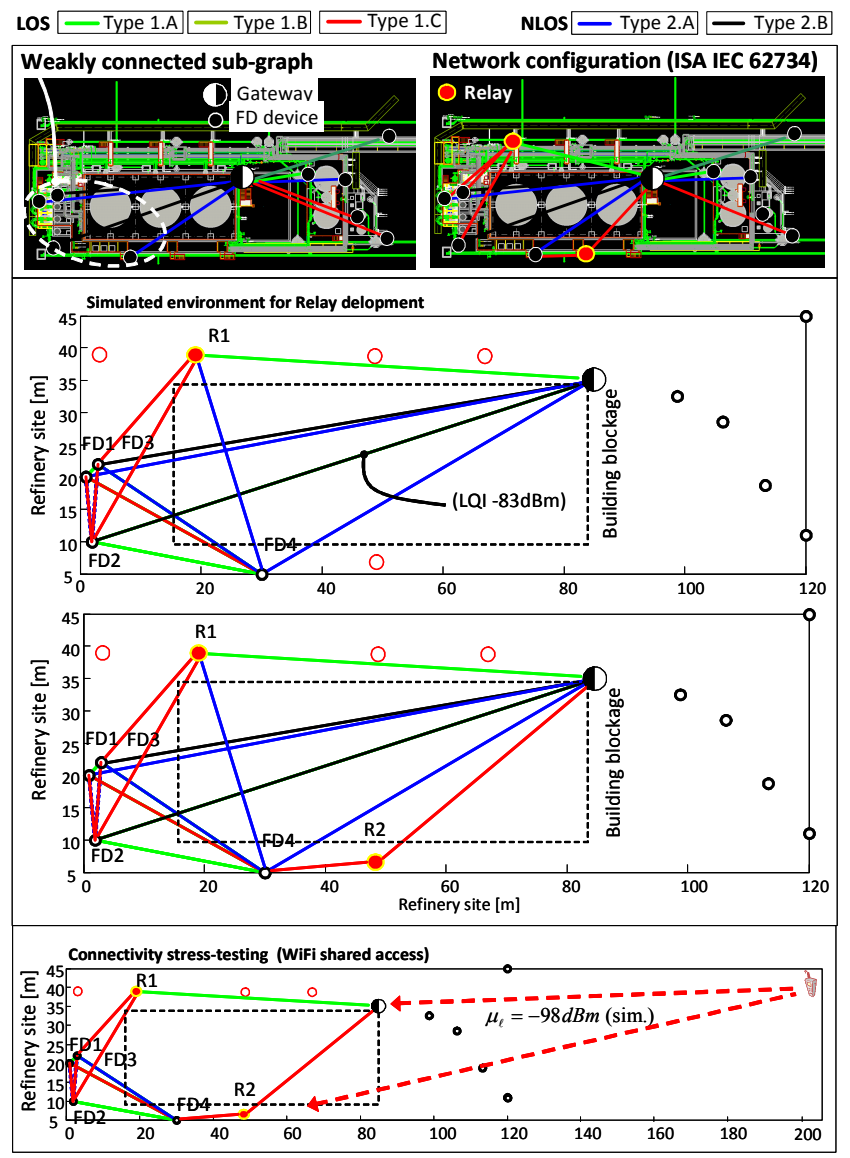

Fig. 9. Deployment case study: reference layout for the $(120 \mathrm{~m} \times 45 \mathrm{~m})$ cooling unit site. $N=9$ devices ( 8 FDs and $1 \mathrm{GW}$ ) deployed and $M=5$ relay candidate set points. Bottom figure. Stress-testing of deployment is simulated by a WiFi interference with power $\mu_{\ell}=-98 \mathrm{dBm} . R=2$ relays are deployed to reinforce connectivity.

to be partitioned into two clusters (the FDs are deployed at ground level). A single GW node is deployed to collect data from FDs and re-route them over a Fieldbus network. The FD nodes use a omnidirectional monopole antenna (vertically polarized) while the GW employs a $2 \mathrm{dBi}$ omnidirectional dipole antennas (with $90^{\circ}$ beamwidth). The deployed wireless instruments conform to ISA IEC62734. The Fig. 9 shows the optimal position of the GW to guarantee connectivity with all the FDs: this is located on the staircase behind the cooling structure ( $6 \mathrm{~m}$ from ground level). Connectivity structure and link classification have been obtained by analysis of the 3D CAD model of the plant and validated during postdeployment verification by measurements carried out in the corresponding site (see floor map plant in Fig. 9). Post-layout verification confirms the predicted connectivity structure and the effectiveness of the stochastic model for machine-type links. Although connectivity is observed, additional relay devices are deployed to limit the use of unreliable NLOS links that would penalize the QoS in case of model inaccuracies or co-channel interference.

The corresponding virtual simulated environment is illustrated at bottom of Fig. 9. Here we tackled the problem of reinforcing connectivity focusing on weakly connected 
sub-graph $\mathcal{G}_{s} \subset \mathcal{G}$ of FDs (1-4). Remaining nodes are not considered as critical being connected to the GW by LOS type links with predicted LQI $\hat{g}_{\ell}(8)$ larger than $-58 \mathrm{dBm}$ for all cases and $P_{S} \sim 0.99$. The additional deployment of $R=2$ relay nodes connected with the GW by Type 1 guarantees $\lambda_{2}>1$ and $P_{S} \sim 0.99$ for the two-hop relayed paths

Focusing on node lifetime, the relay R2 monitors $k_{R 2}=6$ links and is the most critical instrument as $\max _{a \in \mathcal{N}} k_{a}=k_{R 2}$. Electric charge is modeled as in (16) assuming a typical active low-power IEEE 802.15.4 PHY transceiver absorbing $19 \mathrm{~mA}$ [20] during transmission (or reception) of an IEEE 802.15.4 data frame of $4 \mathrm{~ms}$ [8] composed by 127 bytes, and reception (or transmission) of the corresponding ACK frame of $1 \mathrm{~ms}$ [8]. Therefore $E_{T-R} \simeq 19 m A \times 4 m s+19 m A \times 1 m s=$ $95 \mu C$ (micro-Coulombs). The additional cost of memory-hold sleep-state is $E_{S}=25 \mu C$ [20]. Using (16), the electric charge subtracted to battery by relay $\mathrm{R} 2$ on every activity cycle is thus $E_{R 2}=k_{R 2} E_{T-R}+E_{S}=595 \mu C$. For a battery with capacity $C=8500 \mathrm{mAh}$ (typical for C-cell lithium battery), the lifetime of relay $\mathrm{R} 2$ is predicted as $T_{\text {life }} \simeq 1.6 \mathrm{yr}$., for $T_{\text {cycle }}=1$ sec. activity cycle.

A stress-testing of relay deployment for graph $\mathcal{G}_{s}$ is performed by simulating a fully overlapping $(\eta=1) \mathrm{WiFi}$ interferer acting as bursty disturbance with power $\mu_{\ell}=-98 \mathrm{dBm}$ and $\mathrm{P}_{\mu_{\ell}}=1$. Since $\mu_{\ell} \geq \beta / \beta_{I}=-100 \mathrm{dBm}$ with threshold $\beta_{I}$ modeled as in (11), then connection probability is ruled by SIR according to (9). While LOS Type 1 links are marginally influenced by the additional interference, for NLOS Type 2 links unreliable connectivity is observed with $P_{S} \sim 0.47$. This is due to WiFi disturbance, combined with possible inaccuracies of 3D layouts and positioning. The deployment of the relay nodes guarantees connectivity even in the presence of overlapping interference: the network structure highlighted at bottom of Fig. 9 can be considered as "interference-immune" after stress-testing.

\section{PERFormanCE EVALUATION OF CLOUd NETWORKS IN RANDOM FIELDS}

In this section we model the cloud-based sensor network as a random geometric graph with probabilistic connectivity [31]. Cloud nodes connect to each other with probability $P_{S}\left(\mathcal{C}_{j}\right)$ in (9), now depending on a randomly assigned link type $\mathcal{C}_{j}$, while WiFi interference acts as disturbance in a shared spectrum access scenario. The goal is to highlight fundamental connectivity properties and provide a comprehensive performance evaluation of arbitrary dense sensor cloud network structures for industrial environments fully characterized by their link types. Focus is on connectivity, battery lifetime and coexistence. For any link $\ell \in \mathcal{V}$ of the random graph, class $\ell \in \mathcal{C}_{j}$ is assigned with probability

$$
\mathrm{P}_{\mathcal{C}_{j}}=\operatorname{Pr}\left[\ell \in \mathcal{C}_{j}\right] \forall j=1, \ldots, C
$$

that provides a virtual representation of the obstruction configuration with loss $\sigma_{\ell}$ as in (6). The binary symmetric adjacency matrix [12] $\mathbf{C}(\mathcal{G})$ is now defined as in Sect. 5. Probability function $\mathrm{P}_{\mathcal{C}_{j}}$ serves as macroscopic description of the deployment site complexity and it can be interpreted as an indicator of the spatial density of the building blockage.

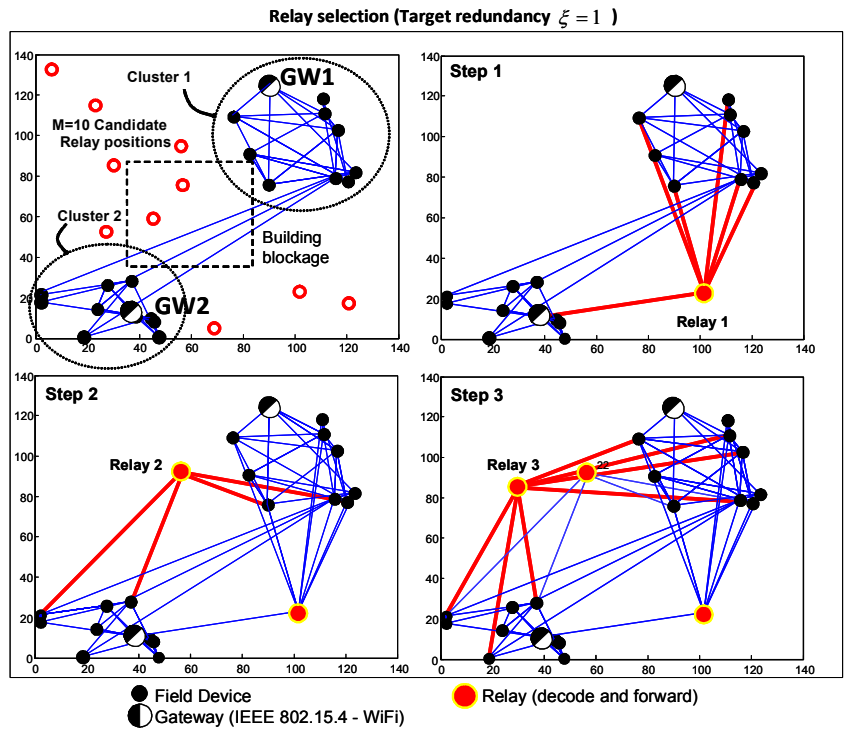

Fig. 10. Iterative relay node deployment example (and connectivity structure) using a reference layout.

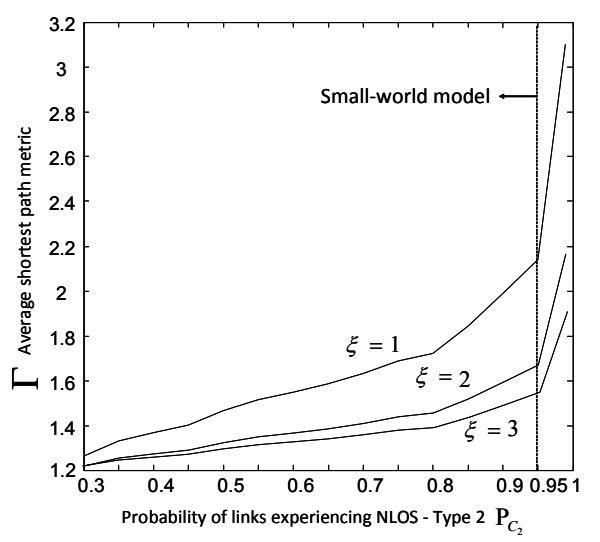

Fig. 11. Average shortest path metric for varying NLOS probability $\mathrm{P}_{\mathcal{C}_{2}}$ and selected target redundancy $\xi$. The cloud network has density 0.02 nodes/sqm and it is deployed on squared field of length $80 \mathrm{~m}$. Small-world model reasonably holds for rewiring probability $\mathrm{P}_{\mathcal{C}_{1}}=0.05$ (or $\mathrm{P}_{\mathcal{C}_{2}}=0.95$ ).

The analysis herein is based on the following limiting assumptions: $i$ ) cloud nodes are randomly deployed according to the (homogeneous) Poisson Point Process (PPP), other models can be found in [32]; ii) a simplified industrial environment is considered as characterized by $C=2$ link types modeling near LOS (now indicated by $j=1$ ) and severe $\operatorname{NLOS}(j=2)$ propagation, respectively ${ }^{6}$; iii) link types are independent and identically distributed according to $(18) ; i v$ ) deployment stresstesting in the shared spectrum access scenario assumes that interference is acting as disturbance with same power for all cloud nodes. This is equivalent to assume that the sensor cloud is localized in a small area (of size in the order of $100 \mathrm{~m}$ ) with WiFi interferer located far enough, so that differences due to path loss are negligible [33]. ${ }^{6}$ The approach can be easily extended for an arbitrary number of link
types, however we found that this choice provides a reasonably accurate
representation of the wireless connectivity in the considered plant scenarios. 


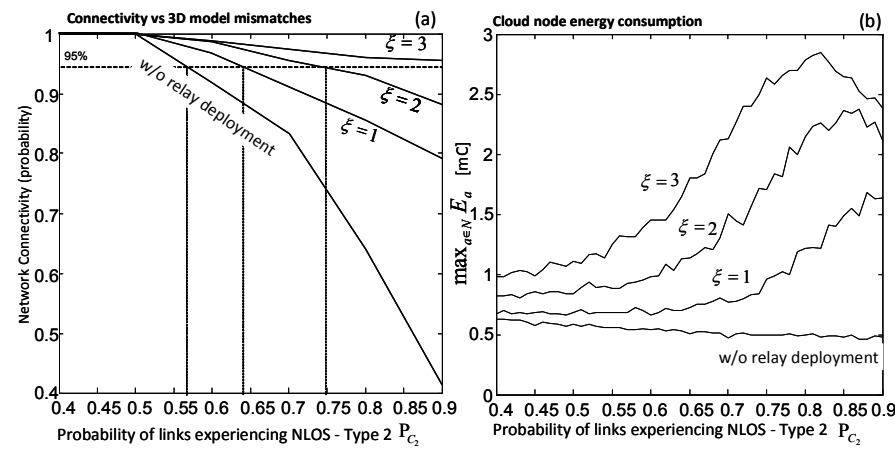

Fig. 12. (a) Connectivity $\operatorname{Pr}\left[\lambda_{2}>0\right]$ under random 3D model inaccuracies, for varying NLOS probability and selected values of target redundancy $\xi=$ $1,2,3$. (b) Predicted node energy consumption $(\mathrm{mC})$ for the same values of $\xi$

In what follows, the iterative relay node deployment approach is illustrated using a reference layout: $N=18$ FDs and 2 GWs are deployed in a squared random field of $150 \mathrm{~m} \times 150 \mathrm{~m}$ characterized by the connectivity structure highlighted in Fig. 10. The network consists of $q=2$ weakly connected clusters: the first one (Cluster 1) contains the GW 1 and 9 FDs $\mathcal{N}_{1} \subset \mathcal{N}$ with $\left[\mathbf{v}_{2}\right]_{i \in \mathcal{N}_{1}}<0$. Cluster 2 (corresponding to nodes $\mathcal{N}_{2} \subset \mathcal{N}$ with $\left[\mathbf{v}_{2}\right]_{i \in \mathcal{N}_{2}}>0$ ) is weakly connected with $\mathcal{N}_{1}$ and contains the $\mathrm{GW}$ node 2. At first iteration, the relay is chosen among $M=10$ candidate positions to provide a secondary path to connect GW 2 with the cluster $\mathcal{N}_{1}$ (see highlighted links). The new graph $\mathcal{G}^{(1)}$ of $N+1$ devices has now $\lambda_{2}\left(\mathcal{G}^{(1)}\right)=0.45$. At second and third steps, 2 new relays are chosen to connect pairs of FDs belonging to cluster $\mathcal{N}_{1}$ and $\mathcal{N}_{2}$, respectively. The third relay provides $\lambda_{2}\left(\mathcal{G}^{(3)}\right)=1.14$ while clusters $\mathcal{N}_{1}$ and $\mathcal{N}_{2}$ are now connected by at least 4 disjoint paths. To conclude, for the considered topology the additional deployment of $R=3$ relay nodes guarantees reliable connectivity as $\lambda_{2}\left(\mathcal{G}^{(3)}\right)>1$ with $\xi=1$ being the assigned target redundancy.

\section{A. Small-world connectivity and node lifetime}

The heterogeneity of link types ranging from near LOS (with probability $\mathrm{P}_{\mathcal{C}_{1}}$ ) to severe NLOS (with probability $\mathrm{P}_{\mathcal{C}_{2}}=1-\mathrm{P}_{\mathcal{C}_{1}}$ ) makes the connectivity structure to be reasonably described by a small world model where the fraction $\mathrm{P}_{\mathcal{C}_{1}}$ of near LOS links can be interpreted as rewiring probability [12]. To verify this property, in Fig. 11 the average shortest path metric $\Gamma$ defined in (17) is computed for varying probability of NLOS links $\mathrm{P}_{\mathcal{C}_{2}}$ and target redundancy $\xi$. The considered cloud network has initial density of 0.02 nodes $/ \mathrm{m}^{2}$ (before relay deployment), in a squared field of length $80 \mathrm{~m}$ : additional relays are chosen from up to a maximum of $M=150$ candidate points and deployed to guarantee the corresponding redundancy target $\xi$. For the considered cases, a phase transition in the connectivity behavior exists for $\mathrm{P}_{\mathcal{C}_{1}}=5 \%$ (or $\mathrm{P}_{\mathcal{C}_{2}}=95 \%$ ) while for $\mathrm{P}_{\mathcal{C}_{1}}>5 \%$ smallworld phenomena emerge. Small-world connectivity causes an immediate drop of average shortest path metric (as $\Gamma<2$ ), thus simplifying the layout design for connectivity robustness in delay-sensitive applications.

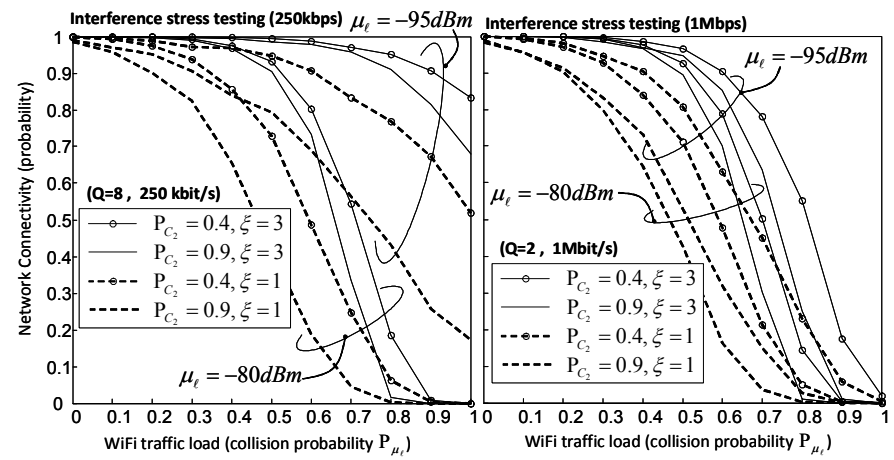

Fig. 13. Interference stress-testing: connectivity probability for varying WiFi traffic loads and for selected values of received power, NLOS probability and target redundancy. Stress-testing of connectivity also includes the case for enhanced PHY data rate (on the right).

In Fig. 12-(a) we now analyze the connectivity probability $\operatorname{Pr}\left[\lambda_{2}>0\right]$ subject to $2 \mathrm{D}$ model inaccuracies, for varying fraction of NLOS links $\mathrm{P}_{\mathcal{C}_{2}}$ and redundancy $\xi=1,2,3$ for relay deployment. Considering propagation fields characterized by a high fraction of NLOS type links $0.75 \leq \mathrm{P}_{\mathcal{C}_{2}} \leq 0.9$, the target redundancy design parameter for $99 \%$ connectivity should be set to $\xi=3$. For mild propagation environments characterized by $0.64 \leq \mathrm{P}_{\mathcal{C}_{2}}<0.75$ a reasonable value for the target redundancy (to avoid unnecessary energy costs) is $\xi=2$. As depicted in Fig. 12-(b), connectivity robustness is guaranteed at the cost of lower node battery lifetime, as energy consumption increases with $\xi$. The figure shows the predicted maximum node energy cost for the same design parameters $\xi$ and probability $\mathrm{P}_{\mathcal{C}_{2}}$ (modeling of consumption is given in Sect. 5.B).

\section{B. Interference stress-testing}

In Fig. 13 the perturbation of connectivity probability $\operatorname{Pr}\left[\lambda_{2}>0\right]$ and graph structure due to a full-overlapping $(\eta=$ 1) WiFi interference is analyzed for varying traffic loads $P_{\mu_{\ell}}$, NLOS probability, target redundancy $\xi=1,3$ and received power $\mu_{\ell}=-80 \mathrm{dBm}$ and $\mu_{\ell}=-95 \mathrm{dBm}$, modeling different WiFi node deployments. Immunity to interference is analyzed versus frame collision $\mathrm{P}_{\mu_{\ell}}$, interference power $\mu_{\ell}$ and design parameter $\xi$, looking for conditions where WiFi activity has no meaningful impact on cloud connectivity. By choosing $\xi=3$, connectivity can be reasonably guaranteed against collision probabilities $\mathrm{P}_{\mu_{\ell}} \leq 0.5$ and $\mathrm{P}_{\mu_{\ell}} \leq 0.3$, for $\mu_{\ell}=-95 \mathrm{dBm}$ and $\mu_{\ell}=-80 \mathrm{dBm}$, respectively. In the same figure (on the right), stress-testing of connectivity is also carried out for IEEE 802.15.4 devices configured to transmit at larger PHY data rate $(1 \mathrm{Mbps})$. The more significant interference capturing effect (Sect. 4) observed when reducing the DSSS factor from $Q_{1}=8$ to $Q_{2}=2$ (in exchange for data-rate increase) limits the maximum tolerable collision probability to $\mathrm{P}_{\mu_{\ell}} \leq 0.3$ and $\mathrm{P}_{\mu_{\ell}} \leq 0.15$, respectively. The use of advanced cooperative spectrum sharing policies (see e.g., [34]) is thus mandatory in high traffic load scenarios. 


\section{CONCLUDING REMARKS}

In this paper we proposed industry-standard methods and tools to support virtual coverage prediction, deployment optimization of industrial wireless cloud networks. A stochastic model for the prediction of machine-type connectivity is proposed and validated by measurements. The model can account for imperfect node positioning and 3D layout inaccuracies or uncertainties. We showed that the wireless links partitioned into LOS/NLOS classes statistically describe the observed propagation loss over short-range communication and given the 3D structure of the obstructions (the position of the industrial equipment and buildings). A separate channel model is thus proposed to predict the QoS for each link-type. Next, techniques and tools for relay deployment and prediction of network performance in interference-limited environments are tailored for industry-standard devices operating according to different PHY layer configurations. The problem of relay deployment is considered as well to improve the reliability of cloud networks characterized by weakly connected clusters of devices with an high percentage of unreliable NLOS links. Experimental results and post-layout verification from the surveys conducted in oil refinery environments confirm the effectiveness of the proposed toolkit as it provides practical solutions for virtual layout design with a degree of accuracy reasonable for industrial network planning.

\section{REFERENCES}

[1] A. Willig, "Recent and emerging topics in wireless industrial communications: a selection," IEEE Trans. on Ind. Informatics, vol. 4, no. 2, May 2008.

[2] D. Miorandi, S. Sicari, F. De Pellegrini, I. Chlamtac, "Internet of things: vision, applications and research challenges," Elsevier Ad Hoc Networks, vol. 10, Issue 7, pp. 1497-1516, September 2012.

[3] D. Zuehlke, "Smart factory - towards a factory-of-things," Annual Reviews in Control, vol. 34, no. 1, pp. 129-138, April 2010.

[4] A. Alamri, et al., "A survey on sensor-cloud: architecture, applications, and approaches," International Journal of Distributed Sensor Networks, Hindawi Publ., vol. 2013, Article ID 917923, 18 pages, 2013.

[5] J. Sýkora and H. Mark, "Dense Cooperative Wireless Cloud Network (DIWINE)", in Proceedings of the Future Network and Mobile Summit (FuNeMS), 3-5 July 2013.

[6] S. Savazzi, M. Nicoli, F. Carminati, M. Riva, "A Bayesian approach to device-free localization: modeling and experimental assessment," IEEE Journal on Sel. Topics in Signal Proc., (available in pre-print), February 2014.

[7] L. Atzori, A. Iera, G. Morabito, "SIoT: Giving a Social Structure to the Internet of Things," IEEE Communications Letters, vol.15, no.11, pp.1193,1195, November 2011.

[8] S. Petersen, S. Carlsen, "WirelessHART versus ISA100.11a: the format war hits the factory floor," IEEE Ind. Electronics Mag., vol. 5, no.4, pp. 23,34, Dec. 2011.

[9] G. Ghielmini, at al., "Virtual factory manager for semantic data handling," CIRP Journal of Manufacturing Science and Technology, vol. 6, no. 4, pp. 281-291, 2013, ISSN 1755-5817.

[10] Y. Zhou, et al., "Guest Editorial: Virtual MIMO," IEEE Journal on Selected Areas in Communications, vol.31, no.10, October 2013.

[11] S. Savazzi, S. Guardiano, U. Spagnolini, "Wireless sensor network modeling and deployment challenges in oil and gas refinery plants" International Journal of Distributed Sensor Networks, Hindawi vol. 2013, Article ID 383168, p.17, 2013.

[12] S. Boccaletti, et al. "Complex networks: structure and dynamics,"Elsevier Physics Reports no. 424, pp. 175 - 308, 2006.

[13] Recommendation ITU-R P.1411-6 "Propagation data and prediction methods for the planning of short-range outdoor radio communication systems and radio local area networks in the frequency range $300 \mathrm{MHz}$ to $100 \mathrm{GHz}$," P. Series Radiowave propagation, ITU, Feb. 2012.
[14] J. Lei, L. Greenstein, R. Yates, "Link gain matrix estimation in distributed wireless networks," Proc. of IEEE GLOBECOM, New Orleans, USA, Dec. 2008.

[15] N. Baccour, at al. "Radio link quality estimation in wireless sensor networks: a survey," ACM Transactions on Sensor Networks, vol. 8 no. 4, September 2012

[16] S. R. Saunders, A. A. Zavala, Antennas and Propagation for Wireless Communications Systems, Wiley, 2nd edition, May 2007.

[17] H. Mokhtaru, P. Lazaridis, "Comparative study of lateral profile knifeedge diffraction and ray tracing technique using GTD in urban environment," IEEE Trans. on Vehicular Tech., vol. 48, no. 1, Jan. 1999.

[18] W. Guo; W. M. Healy, Z. MengChu, "Impacts of 2.4-GHz ISM band Interference on IEEE 802.15.4 wireless sensor network reliability in buildings," IEEE Trans. on Instr. and Measurement, vol. 61, no. 9, pp. 2533,2544, Sept. 2012.

[19] Dong Yang, Youzhi Xu, and Mikael Gidlund, "Wireless coexistence between IEEE 802.11 and IEEE 802.15.4-based networks: a survey," International Journal of Distributed Sensor Networks, vol. 2011, Article ID 912152, 17 pages, 2011. doi:10.1155/2011/912152.

[20] Data-sheet JN-DS-JN5148-001, IEEE 802.15.4 Wireless Microcontroller JN5148-001, NXP Laboratories, UK, 2012.

[21] Z. Iqbal, et al., "Deterministic and event triggered MAC protocol for industrial wireless networks," Proc. of IEEE International Conference on Industrial Technology (ICIT 2013), Cape Town, South Africa, Feb. 2013.

[22] L. Angrisani, M. Bertocco, D. Fortin and A. Sona, "Experimental study of coexistence issues between IEEE 802.11b and IEEE 802.15.4 wireless networks," IEEE Trans. on Instr. and Measurements vol. 57, no. 8, pp. 1514-1523, Aug. 2008.

[23] D. Camps-Mur, A. Garcia-Saavedra, P. Serrano, "Device-to-Device communications with WiFi direct: overview and experimentation," IEEE Wireless Communications, vol. 20, no. 3, June 2013.

[24] S. Fortunato, "Community detection in graphs," Physics Reports, vol. 486, no. 3-5, pp. 75-174, February 2010.

[25] H. Sundaram, et al., "Understanding community dynamics in online social networks: a multidisciplinary review," IEEE Signal Proc. Mag., vol. 29, no. 2, pp. 33-40, March 2012.

[26] M. E. J. Newman and M. Girvan, "Finding and evaluating community structure in networks," Phys. Rev. E 69, 026113, 2004.

[27] M. Younis and K. Akkaya, "Strategies and techniques for node placement in wireless sensor networks: a survey,'Elsevier Ad-Hoc Networks J., vol 6, no. 4, pp.621-655, 2008.

[28] N. M. Maia de Abreu, "Old and new results on algebraic connectivity of graphs,"Elsevier Linear Algebra and its Applications, vol. 423, no. 1, pp. 53-73, May 2007.

[29] R. Olfati-Saber, R. M. Murray, "Consensus problems in networks of agents with switching topology and time-delays," IEEE Trans. on Automatic Control, vol.49, no.9, pp.1520,1533, Sept. 2004.

[30] T. M. Chiwewe, G. P. Hancke, "A distributed topology control technique for low interference and energy efficiency in wireless sensor networks," IEEE Trans. on Ind. Informatics, vol. 8, no. 1, Feb. 2012.

[31] M. Haenggi, J. G. Andrews, F. Baccelli, O. Dousse, M. Franceschetti, "Stochastic geometry and random graphs for the analysis and design of wireless networks," IEEE Journal on Selected Areas in Communications, vol.27, no.7, pp.1029-1046, September 2009.

[32] J. G. Andrews, R. K. Ganti, M. Haenggi, N. Jindal, S. Weber, "A primer on spatial modeling and analysis in wireless networks," IEEE Communications Magazine, vol.48, no.11, pp.156-163, November 2010.

[33] E. Axell, G. Leus, E. G. Larsson, H. V. Poor, "Spectrum sensing for cognitive radio: state-of-the-art and recent advances," IEEE Signal Processing Magazine, vol.29, no.3, pp.101-116, May 2012.

[34] O. Simeone, et. al. "Spectrum leasing to cooperating secondary ad hoc networks," IEEE Journal on Selected Areas in Communications, Cognitive Radio: Theory and Applications, vol. 26, no. 1, January 2008. 